\title{
THE DEEP2 GALAXY REDSHIFT SURVEY: THE VORONOI-DELAUNAY METHOD CATALOG OF GALAXY GROUPS
}

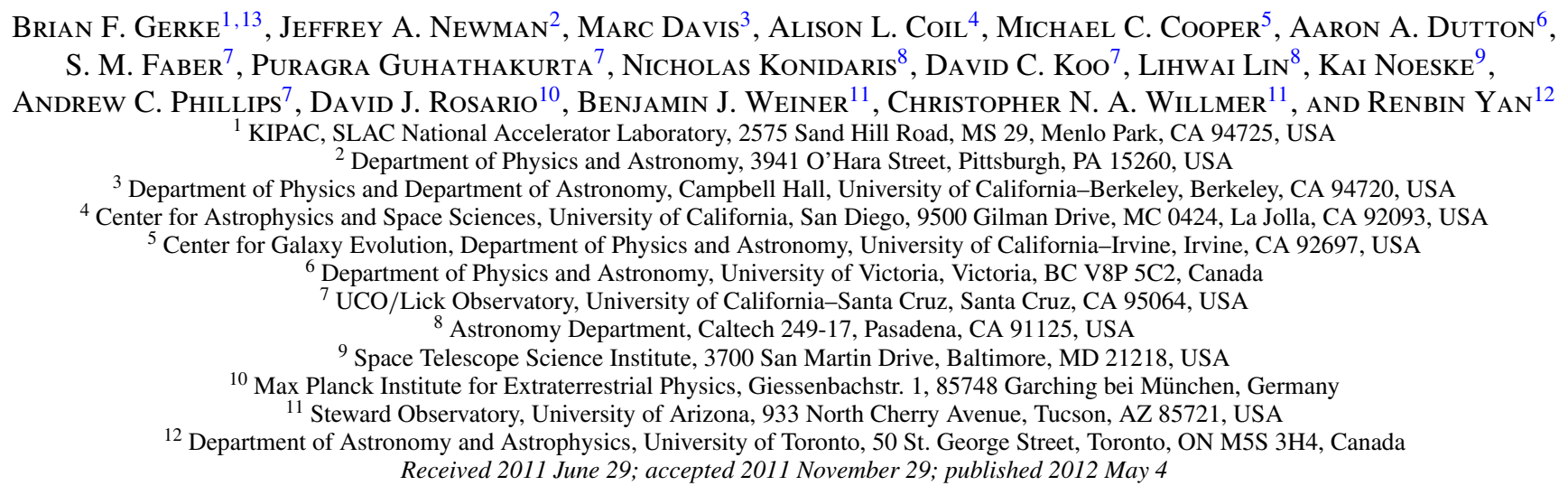

\begin{abstract}
We present a public catalog of galaxy groups constructed from the spectroscopic sample of galaxies in the fourth data release from the Deep Extragalactic Evolutionary Probe 2 (DEEP2) Galaxy Redshift Survey, including the Extended Groth Strip (EGS). The catalog contains 1165 groups with two or more members in the EGS over the redshift range $0<z<1.5$ and 1295 groups at $z>0.6$ in the rest of DEEP2. Twenty-five percent of EGS galaxies and fourteen percent of high- $z$ DEEP2 galaxies are assigned to galaxy groups. The groups were detected using the Voronoi-Delaunay method (VDM) after it has been optimized on mock DEEP2 catalogs following similar methods to those employed in Gerke et al. In the optimization effort, we have taken particular care to ensure that the mock catalogs resemble the data as closely as possible, and we have fine-tuned our methods separately on mocks constructed for the EGS and the rest of DEEP2. We have also probed the effect of the assumed cosmology on our inferred group-finding efficiency by performing our optimization on three different mock catalogs with different background cosmologies, finding large differences in the group-finding success we can achieve for these different mocks. Using the mock catalog whose background cosmology is most consistent with current data, we estimate that the DEEP2 group catalog is $72 \%$ complete and $61 \%$ pure (74\% and $67 \%$ for the EGS) and that the group finder correctly classifies $70 \%$ of galaxies that truly belong to groups, with an additional $46 \%$ of interloper galaxies contaminating the catalog (66\% and $43 \%$ for the EGS). We also confirm that the VDM catalog reconstructs the abundance of galaxy groups with velocity dispersions above $\sim 300 \mathrm{~km} \mathrm{~s}^{-1}$ to an accuracy better than the sample variance, and this successful reconstruction is not strongly dependent on cosmology. This makes the DEEP2 group catalog a promising probe of the growth of cosmic structure that can potentially be used for cosmological tests.
\end{abstract}

Key words: galaxies: clusters: general - galaxies: high-redshift

Online-only material: color figures, machine-readable tables

\section{INTRODUCTION}

The spherical or ellipsoidal gravitational collapse of an overdense region of space in an expanding background is a simple dynamical problem that can be used as an Ansatz to predict the mass distribution of massive collapsed structures in the cold dark matter cosmological paradigm, as a function of the cosmological parameters (Press \& Schechter 1974; Bardeen et al. 1986; Sheth \& Tormen 2002). This has led to the widespread use of galaxy clusters and groups as convenient cosmological probes. In addition, it has long been apparent that the galaxy population in groups and clusters differs in its properties from the general population of galaxies (e.g., Spitzer \& Baade 1951; Dressler 1980) and that the two populations exhibit different evolution (Butcher \& Oemler 1984). This suggests that galaxy groups and clusters can be used as laboratories for studying

\footnotetext{
${ }^{13}$ Current address: Lawrence Berkeley National Laboratory, 1 Cyclotron
} Road, MS 90-4000, Berkeley, CA 94720, USA. evolutionary processes in galaxies. For both of these reasons, a catalog of groups and clusters has been derived for every large survey of galaxies.

The history of group and cluster finding in galaxy surveys includes a wide variety of detection methods, starting with the visual detection of local clusters in imaging data by Abell (1958). The approaches can be broadly divided into two categories: those that use photometric data only, and those that use spectroscopic redshift information. In relatively shallow photometric data, it is possible to find clusters by simply looking for overdensities in the on-sky galaxy distribution, but in modern, deep photometric surveys, foreground and background objects quickly overwhelm these density peaks at all but the lowest redshifts. Recent photometric cluster-finding algorithms thus typically also rely on assumptions about the properties of galaxies in clusters, on photometric redshift estimates, or on a combination of the two (e.g., Postman et al. 1996; Gladders \& Yee 2000; Koester et al. 2007; Li \& Yee 2008; Liu et al. 2008; Adami et al. 
2010; Hao et al. 2010; Milkeraitis et al. 2010; Soares-Santos et al. 2011).

Spectroscopic galaxy redshift surveys remove much of the problem of projection effects from cluster-finding efforts, though not all of it, owing to the well-known finger-of-god effect. Since closely neighboring galaxies in redshift space can be assumed to be physically associated, it is possible to use spectroscopic surveys to reliably detect relatively low-mass, galaxypoor systems (i.e., galaxy groups) in addition to rich, massive clusters. The most popular approach historically has been the friends-of-friends (FoF), or percolation, algorithm, which links galaxies together with their neighbors that lie within a given linking length on the sky and in redshift space, without reference to galaxy properties. This technique was pioneered in the CfA redshift survey (Huchra \& Geller 1982) and is still in common use in present-day redshift surveys (e.g., Eke et al. 2004; Berlind et al. 2006; Knobel et al. 2009). Recently, other redshift-space algorithms have also had success by including simple assumptions about the properties of galaxies in clusters and groups (e.g., Miller et al. 2005; Yang et al. 2005). The primary disadvantage of cluster-finding in redshift-space data is that spectroscopic surveys generally cannot schedule every galaxy for observation, leading to a sparser sampling of the galaxy population than is available in photometric data. When the sampling rate becomes extremely low, standard methods like FoF have a very high failure rate. This is a particular concern for high-redshift surveys for which spectroscopy is very observationally expensive.

In any case, since cluster-finding algorithms search for spatial associations in a point-like data set, it can be shown that a perfect reconstruction of the true, underlying bound systems can never be achieved owing to random noise (Szapudi \& Szalay 1996). Indeed, it has long been known that a fundamental tradeoff exists between the purity and completeness of a cluster catalog when compared with the underlying dark-matter halo population in $N$-body models (Nolthenius \& White 1987): a catalog cannot be constructed that detects all existing clusters and is free of false detections. In order to fully understand and minimize these inevitable errors, it has become standard practice to use mock galaxy catalogs, based on $N$-body darkmatter simulations, to test cluster-finding algorithms, optimize their free parameters, and estimate the level of error in the final catalog. In all such studies, some effort has been made to ensure that the mock catalogs resemble the data at least in a qualitative sense, but little work has been done to examine how quantitative differences between the mocks and the data, or inaccuracies in the assumed background cosmology, will impact the groupfinder calibration.

In this paper, we present a catalog of galaxy groups and clusters for the final data release (DR4) of the Deep Extragalactic Evolutionary Probe 2 (DEEP2) Galaxy Redshift Survey (J. A. Newman et al., in preparation), a spectroscopic survey of tens of thousands of mostly high-redshift galaxies, with a median redshift around $z=0.9$. This catalog is made available to the public on the DEEP2 DR4 website. ${ }^{14}$ To construct this catalog, we make use of the Voronoi-Delaunay method (VDM) group finder, which was originally developed by Marinoni et al. (2002) for use in relatively sparsely sampled, high-redshift surveys similar to DEEP2. To test and calibrate our methods, we make use of a set of realistic mock galaxy catalogs that we have recently constructed for DEEP2 (B. F. Gerke et al., in

\footnotetext{
14 http://deep.berkeley.edu/dr4
}

preparation). These catalogs have been constructed for several different background cosmologies, allowing us to test the impact of cosmology on the group-finder calibration and error rate. This work updates and expands upon the group-finding efforts of Gerke et al. (2005, hereafter G05), who detected groups with the VDM algorithm in early DEEP2 data using an earlier set of DEEP2 mocks for calibration.

Our goals in constructing this catalog are similar to the historical ones described above. First, a catalog of galaxy groups is an interesting tool for studying the evolution of the galaxy population in DEEP2, as well as for studying the baryonic astrophysics of groups and clusters themselves, as has been demonstrated in various papers using the G05 catalog (Fang et al. 2007; Coil et al. 2006a; Gerke et al. 2007; Georgakakis et al. 2008; Jeltema et al. 2009). In addition, it has been shown that a catalog of groups from a survey like DEEP2 can be used to probe cosmological parameters, including the equation of state of the dark energy, by counting groups as a function of their redshift and velocity dispersion (Newman et al. 2002); we aim to produce a group catalog suitable for that purpose here.

We proceed as follows. In Section 2, we introduce the DEEP2 data set and describe our methods for constructing realistic DEEP2 mock catalogs with which to test and refine our groupfinding methods. Section 3 details the specific criteria we use for such testing. In Section 4 we give a brief overview of VDM, including some changes to the G05 algorithm, and we optimize the algorithm on our mock catalogs in Section 5. The latter section also explores the dependence of our optimum groupfinding parameters on the assumed cosmology of the mock catalogs. Section 6 presents the DEEP2 group catalog and compares it to other high-redshift spectroscopic group catalogs. Throughout this paper, where necessary and not otherwise specified, we assume a flat $\Lambda$ CDM cosmology with $\Omega_{M}=0.3$ and $h=0.7$.

\section{THE DEEP2 SURVEY AND MOCK CATALOGS}

\subsection{The DEEP2 Data Set}

The DEEP2 Galaxy Redshift Survey is the largest spectroscopic survey of homogeneously selected galaxies at redshifts near unity. It consists of some 50,000 spectra obtained in $1 \mathrm{hr}$ exposures with the DEIMOS spectrograph (Faber et al. 2003) on the Keck II telescope. This data set yielded more than 35,000 confirmed galaxy redshifts; the rest were either stellar spectra or failed to yield a reliable redshift identification. DEEP2 will be comprehensively described in J. A. Newman et al. (in preparation); most details of the survey can also be found in Willmer et al. (2006), Davis et al. (2004), and Davis et al. (2007). Here we summarize the main survey characteristics, focusing on issues of particular importance for group finding.

DEEP2 comprises four separate observing fields, chosen to lie in regions of low Galactic dust extinction that are also widely separated in R.A. to allow for year-round observing. With a combined area of approximately $3 \mathrm{deg}^{2}$, the DEEP2 fields probe a volume of $5.6 \times 10^{6} h^{-3} \mathrm{Mpc}^{3}$ over the primary DEEP2 redshift range $0.75<z<1.4$. This is an excellent survey volume for studying galaxy groups: at the relevant epochs, one expects to find more than 1000 dark-matter halos with masses in the range of galaxy groups (roughly $5 \times 10^{12} M_{\odot} \lesssim M_{\text {halo }} \lesssim 1 \times 10^{14} M_{\odot}$ ) in a volume of this size. DEEP2 is less well suited for studying clusters: at most there should be a few to a few tens of halos with cluster masses $\left(M_{\text {halo }} \gtrsim 1 \times 10^{14} M_{\odot}\right)$ in the DEEP2 fields. Since our final catalog will be dominated by objects that are 
traditionally referred to as groups (rather than clusters) we will use that term throughout this work as a shorthand to refer to both groups and clusters.

DEEP2 spectroscopic observations were carried out using the 1200-line diffraction grating on DEIMOS, giving a spectral resolution of $R \sim 6000$. This yields a velocity accuracy of $\sim 30 \mathrm{~km} \mathrm{~s}^{-1}$ (measured from repeat observations of a subset of targets). Such high-precision velocity measurements make DEEP2 an excellent survey for detecting galaxy groups and clusters in redshift space, which is our strategy here. The velocity errors are substantially smaller than typical galaxy peculiar velocities in groups, so the dominant complication for redshiftspace group finding will be the finger-of-god effect, rather than redshift-measurement error.

Targets for DEIMOS spectroscopy were selected down to a limiting magnitude of $R=24.1$ from three-band $(B R I)$ photometric observations taken with the CFH12k imager on the Canada-France-Hawaii Telescope (Coil et al. 2004b). To focus the survey on typical galaxies at $z \sim 1$ (rather than low- $z$ dwarfs) most DEEP2 targets were also restricted to a region of $B-R$ versus $R-I$ color-color space that was chosen to contain a nearly complete sample of galaxies at $z>0.75$ (Davis et al. 2004). Tests with spectroscopic samples observed with no color pre-selection show that the DEEP2 color cuts exclude the bulk of low-redshift targets, while still including 97\% of galaxies in the range $0.75<z<1.4$ (J. A. Newman et al., in preparation). (At $z>1.4$-in the so-called redshift desert-it is difficult to obtain successful galaxy redshifts because of a lack of spectral features in the observed optical waveband.)

Despite the high completeness of the DEEP 2 color selection at high redshift, there remain a number of observational effects that reduce the sampling density of galaxies in groups and clusters. The simplest is the faint apparent magnitude range of $z \sim 1$ galaxies. DEEP2 is limited to luminous galaxies $(L \gtrsim L *$; Willmer et al. 2006) at most redshifts of interest; even massive clusters will contain a few tens of such galaxies at most. At redshifts near $z=0.9$, DEEP2 has a number density of galaxies $n \sim 0.01$ (J. A. Newman et al., in preparation), corresponding to a fairly sparse galaxy sample with mean intergalaxy separation $s \sim 5 h^{-1} \mathrm{Mpc}$ (comoving units). The DEEP2 group sample will thus be made up of systems with relatively low richnesses.

A further complication arises from the effects of $k$-corrections on high-redshift galaxies, which translate the DEEP2 $R$-band apparent magnitude limit into a an evolving, color-dependent luminosity cut in the rest frame of DEEP2 galaxies. As discussed in detail in Willmer et al. (2006) and Gerke et al. (2007), red-sequence galaxies in DEEP2 will have a brighter absolute magnitude limit than blue galaxies at the same redshift, and this disparity increases rapidly with redshift as the observed $R$ band shifts through the rest-frame $B$ band and into the $U$ band (cf. Figure 2 of Gerke et al. 2007). Galaxies on the red sequence are well known to preferentially inhabit the overdense environments of groups and clusters, and this relation holds at $z \sim 1$ in DEEP2 (Cooper et al. 2007; Gerke et al. 2007). This means that groups and clusters of galaxies in DEEP2 will have a lower sampling density than the overall galaxy population, and the observed galaxy population in groups will be skewed toward more luminous objects.

Further undersampling of DEEP2 group galaxies results from the unavoidable realities of multiplexed spectroscopy. DEEP2 spectroscopic targets were observed using custom-designed DEIMOS slitmasks that allowed for simultaneous observations of more than 100 targets. Although slits on DEEP2 masks could

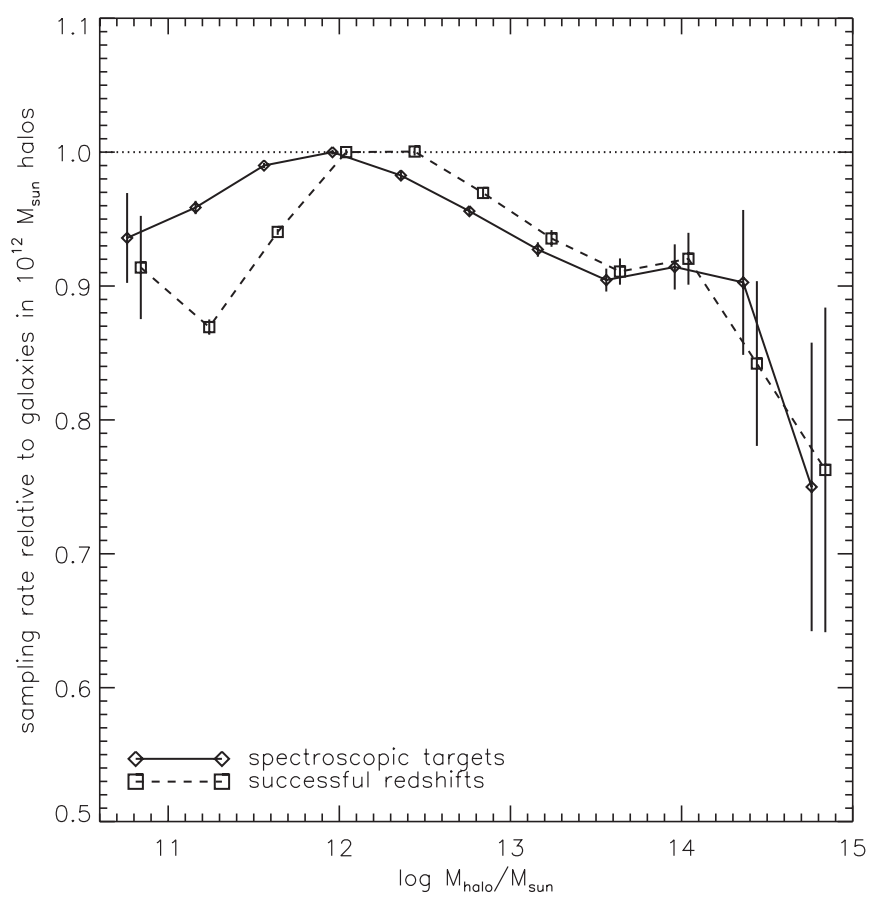

Figure 1. Relative spectroscopic targeting and redshift-success rates of mock DEEP2 galaxies vs. parent halo mass. Because of increased slit conflicts in crowded regions, galaxies in groups and clusters $\left(M_{\text {halo }}>10^{13} M_{\odot}\right)$ are targeted for DEIMOS spectroscopy at a lower rate than galaxies like the Milky Way ( $\left.M_{\text {halo }} \sim 10^{12} M_{\odot}\right)$ (solid line). The effect is mild, however, and remains less than $20 \%$ for all but the most massive clusters in the mock catalog. The sampling rate also falls for low-mass halos, since these contain faint galaxies, some fraction of which fall below the DEEP2 magnitude limit. Faint red galaxies are preferentially undersampled further because they are less likely to yield reliable redshifts; however, this effect is also mild and in any case is limited mostly to galaxies in low-mass halos (dashed line).

be made as short as $3^{\prime \prime}$, and some slits could be designed to observe two neighboring galaxies at once, the requirement that slits not overlap with one another along the spectral direction of a mask inevitably limits the on-sky density of targets that can be observed. Overall, DEEP2 observed roughly $65 \%$ of potential targets, but this fraction is necessarily lower in crowded regions on the sky owing to slit conflicts. The adaptive DEEP2 slitmasktiling strategy relieves crowding issues somewhat, since each target has two chances for selection on overlapping slitmasks, but there is still a distinct anticorrelation between targeting rate and target density: the sampling rate for targets in the most crowded regions on the sky is roughly $70 \%$ of the median sampling rate (G05; J. A. Newman et al., in preparation).

Nevertheless, as discussed in G05, the significant line-of-sight distance covered by DEEP2 means that high-density regions on the sky do not necessarily correspond to high-density regions in three-space. The impact of slit conflicts on the sampling of groups and clusters should therefore be lower than the effect seen in crowded regions of sky. We can test this explicitly using simulated galaxies in the mock catalogs described in Section 2.2. Galaxies in the mocks are selected using the same slitmaskmaking algorithm as used for DEEP2, and since the mocks also contain information on dark-matter halo masses, it is possible to investigate the effect of this algorithm on the sampling rate in group-mass and cluster-mass halos. As shown in Figure 1, galaxies in massive halos are undersampled relative to field galaxies, but the effect is modest, amounting to less than a $10 \%$ reduction in sampling rate at group masses and only a $\sim 20 \%$ reduction for the most massive clusters in the mocks. 
Redshift failure is a final factor that impacts the sampling rate of groups and clusters. After visual inspection, roughly $30 \%$ of DEEP2 spectra fail to yield a reliable redshift (i.e., do not receive DEEP2 redshift quality flag 3 or 4 , which correspond to $95 \%$ and $99 \%$ confidence in the redshift identification, respectively). These redshift failures are excluded from all samples used for group finding. Follow-up observations (C. Steidel 2003, private communication) show that roughly half of these redshift failures lie at $z>1.4$, but the remainder serve to further reduce the DEEP2 sampling rate in the target redshift range. The redshift failure rate increases sharply for galaxies in the faintest half-magnitude of the sample, and it is also boosted for red galaxies, since these tend to lack strong emission lines, making redshift identification more difficult. One might expect that this would decrease the sampling rate preferentially in groups and clusters, which should have a large number of faint red satellite galaxies. It is also possible to test this with the mock catalogs (which account for the color and magnitude dependence of the incompleteness, as discussed in the next section). As shown in Figure 1 (dashed curve), redshift failures have a stronger effect on mock galaxies in low-mass halos (since they preferentially host faint galaxies) than in groups and clusters so that, if anything, the relative sampling rate of groups and clusters is boosted slightly by redshift failures.

In any case, Figure 1 demonstrates the importance of having realistic mock catalogs on which to calibrate group-finding methods. Without accurate modeling of the selection probability for galaxies in massive halos relative to field galaxies, it will be difficult to have confidence in measures of group-finding success (e.g., the completeness and purity of the group catalog). In the next section, we will describe the mock catalogs we use to test our group-finding methods and optimize them for the DEEP2 catalog, focusing on the steps that have been taken to account for all of the different DEEP2 selection effects discussed above.

\subsubsection{The Extended Groth Strip}

Before we proceed, it is important to describe the somewhat different selection criteria that were used in one particular DEEP2 field, the Extended Groth Strip (EGS). This field is also the site of AEGIS, a large compendium of data sets spanning a broad range of wavelengths, from X-ray to radio (Davis et al. 2007). To maximize the redshift coverage of these multiwavelength data sets, DEEP2 targets were selected without color cuts, so that galaxy spectra are obtained across the full redshift range $0<z<1.4$. However, spectroscopic target selection used a probabilistic weighting as a function of color to ensure roughly equal numbers of targets at low and high redshifts; this means that the sampling rate of galaxies will vary differently with redshift than would be expected in a simple magnitude-limited sample. Furthermore, the EGS was observed with a different spectroscopic targeting strategy, so that each galaxy has four chances to be observed on different overlapping slitmasks. The overall sampling rate in the EGS is thus boosted somewhat relative to the rest of DEEP2. These differences in selection mean that it will be important to calibrate our group-finding techniques separately for the EGS and the rest of the DEEP2 sample. Our mock catalogs will therefore need to be flexible enough to account for the differences in selection between the EGS and the rest of DEEP2.

\subsection{DEEP2 Mock Catalogs}

The success of any group finder will depend sensitively on the selection function of galaxies in halos of different masses, since this drives the observed overdensity of groups and clusters relative to the background of field galaxies. It is therefore crucial to test and optimize group-finding algorithms on simulated galaxy catalogs that capture and characterize this mass-dependent selection function as accurately as possible. It is helpful to couch this discussion in the terminology of the halo model (e.g., Peacock \& Smith 2000; Seljak 2000; Ma \& Fry 2000), particularly the halo occupation distribution (HOD) $\bar{N}(M)$, which is the average number of galaxies meeting some criterion (usually a luminosity threshold) in a halo of mass $M$. What we would like is a mock catalog that correctly reproduces the HOD of observed galaxies, not just the HOD for galaxies above some luminosity cut, in group-mass halos. As discussed below, this will require us to improve upon the mocks we used for the initial DEEP2 group-finding calibration in G05.

In that study, we optimized the VDM group finder using the mock catalogs of Yan et al. (2004, hereafter YWC). Those authors produced mock DEEP2 catalogs from a large-volume $\mathrm{N}$-body simulation by adding galaxies to dark-matter halos according to a conditional luminosity function $\Phi(L \mid M)$ whose form and parameters were chosen to be consistent with the Coil et al. (2004a) galaxy autocorrelation function measured in early DEEP2 data. Since the HOD is directly linked to the correlation function, this implied that the HOD in the mocks was consistent with existing data. However, the agreement between the high-redshift mock and measured correlation functions was marginal at best, and later measurements (Coil et al. 2006b) narrowed the error bars on the DEEP2 correlation function so that the existing mocks no longer agree with the data at high redshift. Indeed, direct modeling of the HOD from the DEEP2 correlation function (Zheng et al. 2007) is quite inconsistent with the HOD that was used in YWC. In particular, the YWC HOD had a power-law index of $\sim 0.7$ at high masses, while the HOD derived from DEEP2 data has a power-law index near unity. This suggests that the galaxy occupation of groups in the YWC mocks is quite different from that in the real universe.

Another difficulty arises when we consider color-dependent selection effects. As discussed above, the DEEP2 magnitude limit translates into an evolving, color-dependent luminosity cut that also evolves with redshift, which may lead to preferential undersampling of groups and clusters. This is further complicated by the fact that the color-density relation also evolves over the DEEP2 redshift range (Gerke et al. 2007; Cooper et al. 2007). Correct modeling of galaxy colors in the mock catalogs is therefore critical to proper calibration of our cluster-finding efforts. Unfortunately, the YWC mocks did not contain any color information, so any preferential color-dependent undersampling of groups and clusters was not reflected there. Gerke et al. (2007) addressed this problem by adding colors to the YWC mocks according to the measured DEEP2 color-density relation from Cooper et al. (2006), but this did not address the inaccuracy of the underlying HOD.

A final possible problem involves the choice of cosmological background model used to construct the mock catalogs. The YWC mocks we used in G05 used $N$-body simulations calculated in a flat, $\Lambda \mathrm{CDM}$ cosmology with parameters $\Omega_{M}=0.3$ and $\sigma_{8}=0.9$, both of which lie outside the region of parameter space preferred by current data. Because changing these parameters has a significant impact on the halo abundance at $z \sim 1$, and because any realistic mock catalog will be constrained to match the abundance of galaxies, changes in the cosmology will necessarily have a substantial impact on the HOD. For example, a model with a higher (lower) $\sigma_{8}$ will have a higher (lower) 
Table 1

Summary of the Simulations Used to Construct DEEP2 Mock Catalogs

\begin{tabular}{lcclll}
\hline \hline Simulation & Box Size $^{\mathrm{a}}$ & Fields $^{\mathrm{b}}$ & $\Omega_{M}$ & $\sigma_{8}$ & $h$ \\
\hline Bolshoi & 250 & 40 & 0.27 & 0.82 & 0.7 \\
L160 ART & 160 & 12 & 0.24 & 0.7 & 0.7 \\
L120 ART & 120 & 12 & 0.3 & 0.9 & 0.73 \\
\hline
\end{tabular}

Notes.

a Comoving $h^{-1} \mathrm{Mpc}$ on a side.

b Number of mock $1 \mathrm{deg}^{2}$ DEEP2 fields or $0.5 \mathrm{deg}^{2}$ fields produced from each simulation.

abundance of halos at any given mass, and thus will require a lower (higher) $\bar{N}(M)$ to match the observed galaxy abundance. This effect will be discussed in more depth in the paper describing the new DEEP2 mocks (B. F. Gerke et al., in preparation), but here it will be important to assess its impact on group finding.

Thus, as pointed out in YWC, it is important to update the mock catalogs to match DEEP2 more closely now that a larger data set is available. In this paper, we make use of a new set of DEEP2 mock catalogs that remedy many of the inadequacies of the previous mocks. These mocks will be described in detail in a paper by B. F. Gerke et al. (in preparation); here we summarize the most important improvements over YWC for the purposes of group-finding calibration.

The new mocks are produced from $N$-body simulations that have sufficient mass resolution to detect dark-matter halos and subhalos down to the mass range of dwarf galaxies with absolute magnitudes $\sim M^{*}+10$. This permits us to assign galaxies uniquely to dark-matter halos and subhalos over the full range of redshift and luminosity covered by DEEP2, including the EGS. In order to investigate the impact of different cosmological models on group finding, we have constructed mock catalogs using three different simulations with three different background cosmologies that span the current range of allowed models; these are summarized in Table 1. We use the mocks constructed from the Bolshoi simulation (Klypin et al. 2011) as our fiducial model for quoting our main results, since its parameters are most consistent with current data, but we will use the other two cosmological models to investigate the impact on our results of changes in the cosmological background. As discussed in B. F. Gerke et al. (in preparation), we construct light cones from these simulations, each having the geometry of a single DEEP2 observational field. To properly account for cosmic evolution, we stack different simulation time steps along the line of sight, and we limit the number of lightcones we create for each simulation to ensure that the resulting mocks sample roughly independent volumes at fixed redshift.

To add mock galaxies to these dark-matter-only lightcones, we use the so-called subhalo abundance-matching approach (e.g., Conroy et al. 2006; Vale \& Ostriker 2006) to assign galaxy luminosities to dark-matter subhalos identified in the simulations. Using the measured DEEP2 galaxy luminosity function (including its redshift evolution) and simulated subhalo internal velocity-dispersion function, we map galaxy luminosities to subhalos at fixed number density. By contrast, the dark-matter simulations used for the YWC mocks did not include detections of dark-matter substructures to a sufficiently low mass, so galaxies were assigned to dark-matter halos stochastically from an HOD, with satellite galaxies assigned to randomly selected dark-matter particles. Our subhalo-based procedure should give a more accurate representation of the luminous profiles and galaxy kinematics of galaxy clusters than the Yan et al. (2004) mocks. In addition, the simulations used for the earlier mocks resolved halo masses sufficient to host central galaxies only down to $\sim 0.1 L^{*}$. This made it impossible to create realistic mock catalogs for the EGS field, since this region includes faint dwarf galaxies at low redshifts. Our new mocks resolve halos and subhalos to masses low enough to accommodate all DEEP2 galaxies except for a handful of very faint dwarfs at $z \lesssim 0.05$.

Conroy et al. (2006) showed that the abundance-matching procedure reproduces the galaxy autocorrelation function at a wide range of redshifts, provided that the subhalo velocity function uses the subhalo velocities as measured at the moment they were accreted into larger halos, and for a particular choice of cosmological parameters that is now disfavored by the data. As discussed in B. F. Gerke et al (in preparation), however, for the more accurate cosmology used in Bolshoi, the abundancematching approach does not reproduce the DEEP2-projected two-point function at $z \sim 1$, lying some $20 \%-40 \%$ higher than the measurement from Coil et al. (2006b). As we also discuss in that paper, the likely resolution to this discrepancy would involve an abundance-matching approach that includes scatter in luminosity at fixed subhalo velocity dispersion, with larger scatter at lower dispersion values. This is likely to mainly impact the HOD at low masses, near the transition of $\bar{N}(M)$ between zero and unity, while causing minimal alteration in the HOD at group and cluster masses. Since the Bolshoi mock HOD matches the measured Zheng et al. (2007) HOD well at these masses, we concluded that the clustering mismatch does not preclude using these mocks for group-finder optimization. The overall occupation of group-mass halos in the mocks should represent the real universe well. What then remains is to account for the various observational selection effects that translate this into an observed HOD for groups.

To add galaxy colors to the mocks, we have followed an approach similar to the one used in Gerke et al. (2007; which was itself inspired by the ADDGALS algorithm; R. H. Wechsler et al., in preparation). We assign a rest-frame $U-B$ color to each mock galaxy by drawing a DEEP2 galaxy with similar redshift, luminosity, and local galaxy overdensity. While performing the color assignment, we must also account for galaxies that fall below the DEEP2 apparent magnitude limit. At fixed redshift, there is some luminosity range in which the DEEP2 sample is partially incomplete, depending on galaxy color. In these luminosity ranges, we select galaxies for exclusion from the mock catalog depending on their local density, until the local density distribution in the mock is consistent with the measured distribution in DEEP2. This technique effectively uses local galaxy density as a proxy for color and ensures that the impact of the DEEP2 selection function on the sampling of galaxy environment is accurately reproduced in the mocks. Full details of the color-assignment algorithm (which are somewhat complex and beyond the scope of this discussion) can be found in the paper describing the mock catalogs (B. F. Gerke et al., in preparation).

After assigning rest-frame colors, we then assign observed apparent $R$-band magnitudes by inverting the $k$-correction procedure of Willmer et al. (2006); this procedure accurately reproduces the evolving, color-dependent luminosity cut that is imposed by the DEEP2 magnitude limit, as well as the color-density relation, so any undersampling of groups and clusters owing to color-dependent selection effects should also be captured in these mocks.

As we did in G05 to simulate the effects of DEEP2 spectroscopic target selection, we pass our mock catalogs through 
the same slitmask-making algorithm that was used to schedule objects for DEEP2 observations (Davis et al. 2004; J. A. Newman et al., in preparation). The DEEP2 color cuts do not give a completely pure sample of high-redshift galaxies, so the pool of mock targets for maskmaking also includes foreground $(z<0.75)$ and background $(z>1.4)$ galaxies, as well as randomly positioned stars, in proportions that are consistent with those found in the DEEP2 sample. To make mocks of the EGS field, we use the somewhat different target-selection algorithm that was used for the EGS, including galaxies at all redshifts, but giving higher selection probability to galaxies at $z>0.75$ in a manner that reflects the color-dependent weighting applied to the real EGS. Any density-dependent effects on the sampling rate that are driven by slit conflicts should therefore be fully accounted for in the mocks.

As a final step, we must replicate the effects of DEEP2 redshift failures, as a function of galaxy color and magnitude. To do this, we utilize the incompleteness-correction weighting scheme devised by Willmer et al. (2006). This scheme assigns a weight to each galaxy according to the fraction of similar galaxies (in observed color-color-magnitude space) that failed to yield a redshift. When we add colors to the mock galaxies by selecting galaxies from the DEEP2 sample, we also assign each mock galaxy the incompleteness weight $w_{i}$ of the DEEP2 galaxy we have drawn (with some small corrections, described in Gerke et al. in preparation). Although this was intended to correct for redshift incompleteness in the data, it can be inverted to produce incompleteness in the mock: after we have selected targets with the DEEP2 slitmask-making algorithm, we reject $\sim 30 \%$ of these targets, with a rejection probability given by $1 / w_{i}$. This procedure naturally reproduces any dependence of the DEEP2 redshift-success rate on galaxy color and magnitude.

These mock catalogs accurately reproduce a wide range of statistical properties of the DEEP2 data set (B. F. Gerke et al., in preparation). Most importantly for group-finding efforts, though, the mocks match (1) the HOD at group masses $\left(M \gtrsim 5 \times 10^{12}\right)$, as measured in Zheng et al. (2007) for several different luminosity thresholds, (2) the evolving color-density relation that was measured in Cooper et al. (2006, 2007), and (3) the redshift distribution of the DEEP2 data. These three points of agreement should be sufficient to ensure that the observed DEEP2 HOD for group-mass halos is accurately reproduced by the mocks. We can thus proceed with confidence in using these mocks to optimize our group-finding techniques.

\subsubsection{The Effects of DEEP2 Selection on the Observed Group Population}

First, though, it will be interesting to use the mocks to investigate the impact of observational effects on the galaxy population of massive halos in DEEP2. (We also explored this in some detail in G05; see Figures 2 and 3 of that paper.) Figure 2 summarizes the impact of the various DEEP2 selection effects on galaxies in massive dark-matter halos in a narrow slice through a mock catalog, which contains the most massive highredshift halo in the mocks (this region is depicted in projection on the sky, before and after selection, as the colored points in the upper left and right panels, respectively). There are three primary selection effects that remove galaxies from the mock sample. In the figure, these selections are depicted visually by vertical lines across the main panel, and galaxies' paths through the selection process are shown by horizontal lines running from left to right, with group-mass halos indicated by gray horizontal bands. First, the DEEP2 $R=24.1 \mathrm{mag}$ limit removes faint galaxies, with red galaxies being excluded at brighter luminosities than blue ones. DEIMOS target selection then removes a random subsample of the remaining galaxies, with some preferential rejection occurring in massive halos. Finally, some galaxies fail to yield redshifts, further diluting the sample. The impact of this dilution on the population of galaxies in groups can be quite strong: the most massive halo shown in the main panel loses some $60 \%$ of its members. It also introduces an added degree of stochasticity into the mass selection of halos. The least-massive halo shown in the figure contains two observed galaxies, and would be identified as a group, while the next most massive halo contains only one observed galaxy, so it would be identified as an isolated galaxy.

The lower panels in Figure 2 show the effect on the mass functions of observed galaxies and groups. DEEP2 selection effects mean that the sample of systems with two or more observed galaxies will only be a complete sample of massive halos at relatively high masses $\gtrsim 5 \times 10^{13} M_{\odot}$. However, the cutoff in the mass selection function for groups is quite broad, owing to the stochastic effects mentioned above, so that even halos with $M<10^{12} M_{\odot}$ have some chance of being identified as groups.

The lower panels also show the effect of DEEP2 selection on the relation between halo mass and observed group velocity dispersion (for systems with two or more galaxies at each stage). As expected, the scatter in this relation increases as we move through the selection process, since the number of galaxies sampling the velocity field is reduced. However, a clear correlation remains between the mass of a halo and the dispersion $\sigma_{\mathrm{v}}^{\mathrm{gal}}$ of its galaxies' peculiar velocities. It should therefore be possible, at least in principle, to use a DEEP2 group catalog to measure the halo mass function and constrain cosmological parameters, as proposed in Newman et al. (2002), provided that the halo selection function imposed by DEEP2 galaxy selection can be understood in detail. In addition, it would be necessary to carefully account for the increased scatter in the $M-\sigma_{\mathrm{v}}^{\mathrm{gal}}$ relation imposed by selection effects. We describe a computational approach to achieving this in the Appendix.

\section{CRITERIA FOR GROUP-FINDER OPTIMIZATION}

\subsection{Group-finding Terminology and Success Criteria}

The aim of our group-finding exercise is to identify sets of galaxies that are gravitationally bound to one another in common dark-matter halos. A perfect group catalog would identify all sets of galaxies that share common halos and classify them all as independent groups, with no contamination from other galaxies, and no halo members missed. Any realistic algorithm for finding groups in a galaxy catalog, however, is subject to various sources of error that cannot be fully avoided, owing largely to incompleteness in the catalog and ultimately to the noise inherent in any discrete process (Szapudi \& Szalay 1996). Any individual type of error can typically be reduced to some extent by varying the parameters of the group finder, but this often comes at the expense of increases in other kinds of error. The classic example of this is the tradeoff between merging neighboring small groups together into spuriously large groups on the one hand and fragmenting large groups into smaller subclumps on the other (Nolthenius \& White 1987).

Because there are inevitably such tradeoffs between various different group-finding errors, it is important to define clearly the criteria by which group-finding success is to be judged and the requirements for an acceptable group catalog. As discussed 


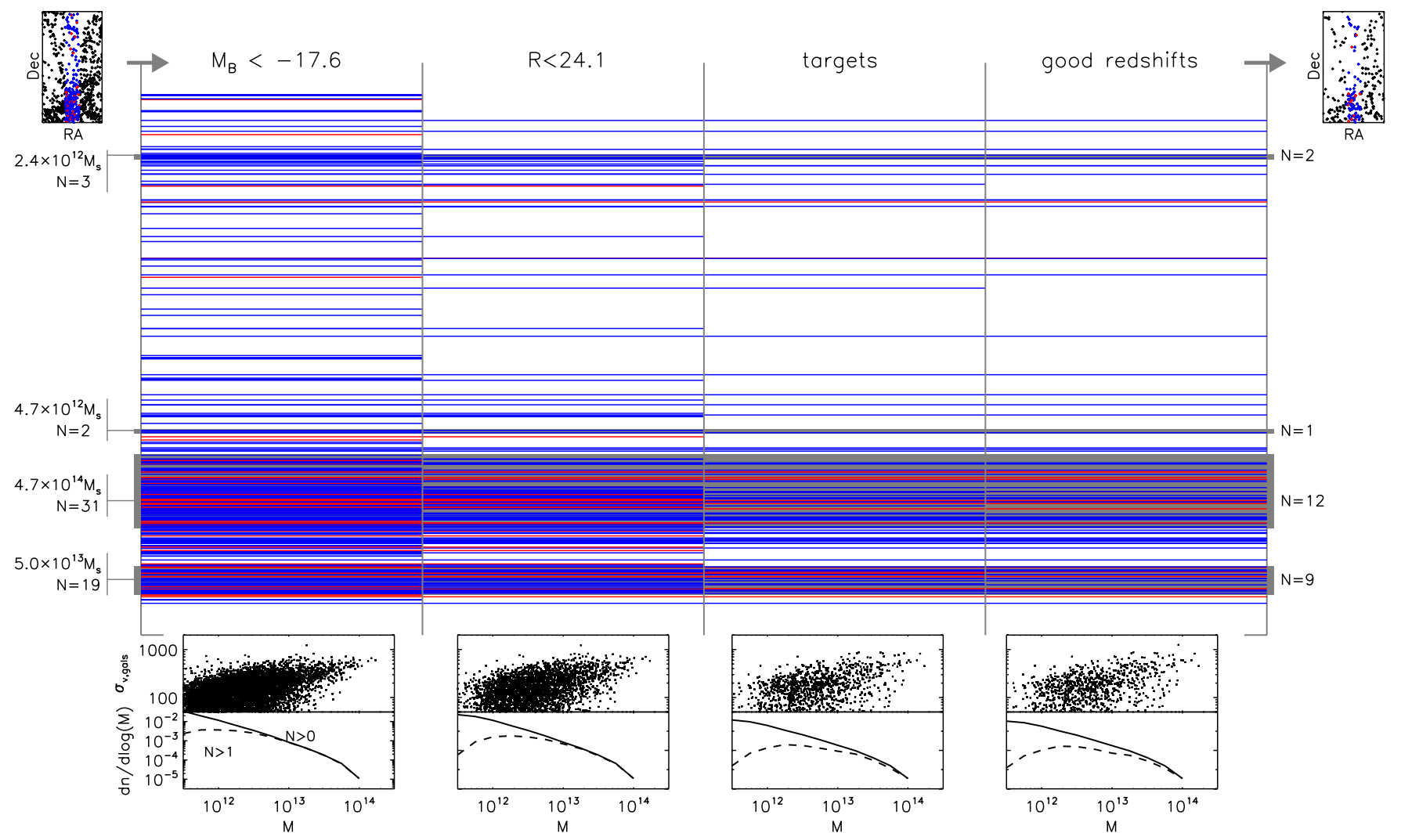

Figure 2. Dilution of a small subregion of a DEEP2 mock catalog by observational selection effects. Galaxies have been selected from a small subregion of a DEEP2 mock catalog, roughly 4 arcmin in R.A. by 30 arcmin in decl. with a redshift depth of 0.05 . This strip is indicated in projection on the sky by the colored points in the panels at top left (before selection) and after selection at top right (afterward). Galaxies on the red sequence (i.e., redder than the red-blue divide given in Willmer et al. 2006) are indicated in red, and blue galaxies are shown in blue. In order to show the impact on cluster selection, this narrow slice in R.A., decl., and redshift was chosen to contain the most massive high-redshift cluster in the mock catalogs (a $4.7 \times 10^{14} M_{\odot}$ object at $z=0.8$ ). The main panel is a schematic diagram of these galaxies' path through the DEEP2 selection process. At left, we begin with horizontal lines (arranged vertically in order of the declination coordinate) representing all galaxies in this subregion more luminous than $M_{B}=-17.6$. Each vertical gray line represents a step in the DEEP2 selection procedure; galaxies are excluded from the sample by the $R=24.1$ apparent magnitude limit by the targeting procedure for assigning galaxies to DEIMOS slits and by failures to obtain good redshifts for some observed galaxies. Horizontal gray bands in the main panel indicate the spatial extent of the four most massive halos in this small region (note that the colored lines within these gray bands are not necessarily all members of these halos, owing to projection effects). The masses of the halos are indicated, as are their richnesses before and after dilution by DEEP2 selection processes. The bottom panels show the aggregate impact of galaxy selection effects on the halo and group population. The lower half of each panel shows the mass function of halos containing one or more galaxy in each sample (solid lines) and the mass function of groups with two or more members (dashed lines). The group population selected in DEEP2 spans a very broad range in mass and represents an incomplete halo sample at all but the very highest masses. The upper half of each panel shows the relation between halo mass and measured group velocity dispersion $\sigma_{\mathrm{v}}^{\text {gal }}$ for all groups with two or more members. The mean relation remains approximately constant, although the scatter increases since there are fewer galaxies sampling the velocity field.

by G05, the optimal balance between different types of error will depend on the particular scientific purpose to be pursued by study of the groups. In the present study, our primary goal is to produce a group catalog that accurately reconstructs the abundance of groups as a function of redshift and velocity dispersion, $N(\sigma, z)$. As discussed in Newman et al. (2002), such a catalog can be used to place constraints on cosmological parameters. Therefore, our optimal group catalog will be the one that most accurately reconstructs $N(\sigma, z)$. It is also of interest to use the group catalog for studies of galaxy evolution in groups (e.g., Gerke et al. 2007) or of the evolution of group scaling relations (e.g., Jeltema et al. 2009); a catalog that can be used for those purposes is a secondary goal. These two goals will drive our choice of metrics for group-finding success in what follows.

\subsubsection{What is a Group?}

In tests using mock catalogs, the "true" group catalog is known, and we are using our group-finding algorithm to produce a "recovered" group catalog; this leads to potential ambiguity in the meaning of the word group. To distinguish clearly between the two cases, we adopt terminology similar to that employed by Koester et al. (2007). For the purposes of discussing group finding in the mocks, a group is defined to be a set of two or more galaxies (the group members) that are linked together by a group-finding algorithm. Galaxies that are not part of any group are called field galaxies. By this definition, a group is not necessarily a gravitationally bound system; rather it is exactly analogous to a group in the real data. By contrast, a halo, for the purposes of discussing group finding, is defined to be a set of galaxies in the observed mock (the halo members) that are all actually bound gravitationally to the same dark-matter halo in the background simulation. ${ }^{15}$ It is possible to have a halo that contains only a single galaxy; such galaxies (and their host halos) are called isolated and are analogous to field galaxies in the group catalog. By comparing the set of groups to the set of

\footnotetext{
15 The assignment of mock galaxies to halos of course depends on the simulation, halo-finding, and mock-making algorithms we employ; we discuss this further in the paper describing the mocks (B. F. Gerke et al., in preparation). For the purposes of this study, though, the galaxy-halo assignment can be taken as "truth," since the choice of algorithms has already been made.
} 
non-isolated halos in the mock catalog, it will then be possible to judge the accuracy of the group finder.

It will also be useful to distinguish between the intrinsic properties of halos (e.g., the total richness, or number of halo members above some luminosity threshold), the observable properties of halos (e.g., the observable richness, or total number of halo members that are in the mock catalog after DEEP2 selection has been applied), and the observed properties of groups (e.g., the observed richness, or total number of group members). Unless otherwise specified, we will always discuss the properties of groups and halos as computed using their member galaxies: for example, the velocity dispersion of a halo will always be the dispersion of the halo members' velocities, $\sigma_{\mathrm{v}}^{\mathrm{gal}}$, rather than the dispersion of the dark-matter particles, $\sigma_{\mathrm{v}}^{\mathrm{DM}}$, unless we explicitly specify that we are talking about a darkmatter dispersion.

\subsubsection{Success and Failure Statistics: Basic Definitions}

There are two primary modes of group-finding failure for which we will adopt the same terminology used in G05. Fragmentation occurs when a group contains a proper subset of the members of a given halo, while overmerging refers to a case in which a group's members include members of more than one halo. A special case of overmerging involves isolated galaxies that are spuriously included in a group; such galaxies are called interlopers. It is also possible for fragmentation and overmerging to occur simultaneously, as when a group contains proper subsets of several different halos.

Fragmentation and overmerging are generally likely to lead to a wide diversity of errors when a group catalog is considered on an object-by-object basis, so it will be useful to define a set of statistics that summarize the overall quality of the catalog. Here we will adopt the statistics used in G05 (with one addition, $f_{\text {noniso }}$, which can be summarized as follows. On a galaxyby-galaxy level, we define the galaxy success rate $S_{\text {gal }}$ to be the fraction of non-isolated halo members that are identified as group members. Conversely, the interloper fraction $f_{\text {int }}$ is the fraction of identified group members that are actually isolated galaxies. It is also worth considering the quality of the field galaxy population, since a perfect group finder would leave behind a clean sample of isolated galaxies. We therefore also compile the non-isolated fraction $f_{\text {noniso, }}$, which is the fraction of field galaxies that are actually non-isolated halo members. On the level of groups and halos, we define two different statistics. Broadly speaking, the completeness $C$ of a group catalog is the fraction of non-isolated halos that are detected as groups, while the purity $P$ is the fraction of groups that correspond to nonisolated halos. In general, the classic tradeoffs inherent in group finding are evident in these statistics: changes to the group finder that improve completeness or galaxy success will typically have negative effects on purity and interloper fraction.

Attentive readers will notice here that we have not yet defined what it means for a halo to be "detected" or for a group to "correspond" to a halo, so the meanings of the terms completeness and purity are still unclear. These definitions, which are somewhat subtle, are the subject of the following sections.

\subsubsection{Matching Groups and Halos}

In order to compute the completeness and purity of a group catalog we must first determine a means for drawing associations between groups and halos. In the case of groups identified in a mock galaxy catalog, the most natural way to do this is to consider the overlap between the groups' and halos' members. This basic approach has been used with good success in many previous studies (e.g., Eke et al. 2004; G05; Koester et al. 2007; Knobel et al. 2009; Cucciati et al. 2010; SoaresSantos et al. 2011). We associate each group to the non-isolated halo that contains a plurality of its members, if any such halo exists (otherwise the cluster is a false detection). Similarly, we associate each non-isolated halo to the group that contains a plurality of its members (again if any such group exists). In the case of ties, e.g., when two halos contribute an equal number of galaxies to a group (an example of overmerging), we choose the object that contains the largest total number of galaxies, or, if this is still not unique, the one with the largest observed velocity dispersion. ${ }^{16}$ Hereafter, we will use the term largest associated object (LAO) to refer to the group (halo) that contains the plurality of a given halo's (group's) members.

This matching procedure is rather lenient and is by no means unique: a group can in principle be associated with a halo with which it shares only a single galaxy, multiple groups can be matched to the same halo (and vice versa), and a cluster may be associated with a halo that is itself associated with some other cluster. For example, if a halo $H$ with five members is divided into two groups, $G_{1}$ with three members and $G_{2}$ with two, then $G_{1}$ and $G_{2}$ are both associated with $H$, but $H$ is only associated with the larger of the two groups, $G_{1}$ (see Figure 4 of G05 or Figure 3 of Knobel et al. 2009 for depictions of other complicated associations). This example also illustrates the difference between one-way and two-way associations: $G_{1}$ is associated with $H$, and vice versa, so this is a two-way match; however, $G_{2}$ is associated with $H$, but the reverse is not true, so this is a one-way match.

In G05, we used a more stringent matching criterion that made an association only when the LAO contained more than $50 \%$ of the galaxies in a given group or halo. This definition has the virtue of removing the need to break ties between possible LAOs, but it is somewhat problematic in the case of low-richness systems. If, for example, a halo containing four galaxies had two of its members assigned to the same group by the group finder, with the other two being called field galaxies, the G05 criterion would class the group as a successful detection but would deem the halo to be undetected. Because of situations like this, we choose here to separate questions of simple group detection from issues of group-finding accuracy. In order to assess the latter, we also compute the overall matching fraction $f$ of each group-halo association: the fraction of galaxies in a given system (group or halo) that is contained in its LAO. In what follows, we will use this fraction to consider more and less stringent limits on accuracy when computing completeness and purity statistics.

\subsubsection{Purity and Completeness}

To compute purity and completeness, it will be necessary to define the criteria by which a group-halo association constitutes a "good" match, to be counted toward these statistics. In general, we will count associations above some threshold in $f$, and we will compute separate purity and completeness values for one-way and two way matches. We will represent these various purity and completeness statistics using the symbols ${ }^{w} P_{f}$ and ${ }^{w} C_{f}$, where we are only counting associations with match fractions larger 16 We would choose randomly if both tie-breaker criteria failed, although this
does not occur in practice. 
than $f$, and $w=1$ or $w=2$ indicates that we are counting one-way or two-way associations.

The simplest statistics to use are ${ }^{1} P_{0}$ and ${ }^{1} C_{0}$, which denote the fraction of groups and halos, respectively, that have any associated object whatsoever, regardless of match fraction or match reciprocity. These values are good for getting an overall sense of the group-finder's success at making bare detections of halos, but their usefulness is somewhat limited since, for example, one could achieve ${ }^{1} C_{0}={ }^{1} P_{0}=1$ simply by placing all galaxies into a single enormous group (in this case, all halos would be associated with the group, and the group would be associated with the largest halo). A more useful pair of statistics is ${ }^{2} C_{0}$ and ${ }^{2} P_{0}$, the fractions of halos and groups that have $t w o$ way associations, regardless of match fraction. These tell us the fraction of halos that were detected without being merged with a larger halo and the fraction of groups that are not lesser subsets of a fragmented halo. In the pathological all-inclusive cluster example above, ${ }^{2} P_{0}=1$, but ${ }^{2} C_{0}$ is near zero, indicating a problem.

This also illustrates the usefulness of comparing one-way and two-way completeness and purity statistics for diagnosing problems with a group finder. If ${ }^{1} C_{0}$ is substantially larger than ${ }^{2} C_{0}$, for example, then a significant fraction of detected halos must have been merged into larger systems, so overmerging is a significant problem. Conversely, if ${ }^{1} P_{0}$ is much larger than ${ }^{2} P_{0}$, then there must be substantial fragmentation in the recovered catalog. It will also be interesting to consider completeness and purity statistics using different values for $f$, such as ${ }^{2} C_{50}$ and ${ }^{2} P_{50}$, which were used in G05. As discussed above, however, using more stringent matching-fraction thresholds can give an overly pessimistic impression of the overall detection success. For our main assessment of overall completeness and purity, then, we will use ${ }^{2} C_{0}$ and ${ }^{2} P_{0}$, since these statistics use the broadest possible definition of a "good" match that does not count fragments and overmergers (beyond the largest object in each fragmented or overmerged system) as successes.

\subsubsection{The Velocity Function of Groups}

In addition to considering the detection efficiency of the group finder on a system-by-system basis, for some science applications one may also be interested in various properties of the group catalog as a whole. In the case of DEEP2, it has been shown (Newman et al. 2002) that the bivariate distribution of groups as a function of redshift and velocity dispersion, $d N(z) / d \sigma_{\mathrm{v}}$, can be used to constrain cosmological parameters, since it depends on the volume element $V(z)$ and on the evolving group velocity function $d n(z) / d \sigma_{\mathrm{v}}$, both of which depend on cosmology. Marinoni et al. (2002) and G05 have shown previously that the VDM group finder can accurately reconstruct this distribution in high-redshift spectroscopic surveys.

In this study, we will use the reconstruction of the velocity function as a second measure of group-finding success. After we have optimized the completeness and purity of our group finder, we will further optimize the group finder to reconstruct $d N(z) / d \sigma_{\mathrm{v}}$ as well as is possible without sacrificing completeness or purity. In practice, this boils down to comparing the number counts of groups and halos in bins of $z$ and $\sigma_{\mathrm{v}}$. Since the distribution is quite steep in $\sigma_{\mathrm{v}}$, it will be important to take some care in our choice of binning. We discuss these details below in Section 5.2

\section{THE GROUP-FINDING ALGORITHM}

\subsection{The Voronoi-Delaunay Group Finder}

The VDM group finder is an algorithm for detecting groups of galaxies in redshift space from spectroscopic survey data. It has advantages over the usual FoF approach in very sparsely sampled data sets, when the linking lengths required for FoF group finding become larger than typical group sizes (for a more detailed discussion of this point, see G05). VDM makes use of the local density information that is obtained by computing the three-dimensional Voronoi tessellation and Delaunay mesh of the galaxies in redshift space. The Voronoi tessellation is a unique partitioning of space about a particular set of points (the galaxies in this case) in which each point is assigned to the unique polyhedral volume of space (the Voronoi cell) that is closer to itself than to any other point. The Delaunay mesh is the geometrical dual of the Voronoi tessellation and consists of a network of line segments that link each point to the points in immediately adjacent Voronoi cells. Galaxies that are directly linked by the Delaunay mesh are called first-order Delaunay neighbors, neighbors of neighbors are second-order Delaunay neighbors, and so on.

The VDM algorithm was first described by Marinoni et al. (2002) who showed that it could be used to detect galaxy groups in a DEEP2-like survey. In particular, they showed that the VDM algorithm could be tuned to accurately reconstruct the distribution of groups as a function of velocity dispersion $\sigma_{\mathrm{v}}$ and redshift $z$, above some threshold in $\sigma_{\mathrm{v}}$; this was confirmed by G05, who produced a preliminary DEEP2 group catalog using a version of the VDM algorithm. VDM has also been applied successfully to the VIMOS VLT Deep Survey (VVDS; Cucciati et al. 2010) and zCOSMOS (Knobel et al. 2009) redshift catalogs. Readers are referred to G05 and Marinoni et al. (2002) for detailed descriptions of the algorithm we will be using in this study. Here, we summarize the basic algorithm and the differences from the version we used in G05.

After computing the Voronoi tessellation and Delaunay mesh for a given galaxy sample, the VDM algorithm proceeds in three phases. In Phase I, the galaxies are first sorted in increasing order of their Voronoi cell volume, a time-saving step which ensures that group finding is attempted in very dense regions first. Then, proceeding through this sorted list in order, we consider each galaxy in turn as a "seed" galaxy for a galaxy group, provided that it has not already been assigned to a group. A cylinder ${ }^{17}$ is drawn around each seed galaxy with radius $\mathcal{R}_{\text {min }}$ and length $2 \mathcal{L}_{\text {min }}$. If that cylinder contains any first-order Delaunay neighbors of the seed galaxy, they are deemed to be part of a group, and the algorithm proceeds to Phase II. If no first-order neighbors are found in the Phase I cylinder, no group is detected, and the algorithm proceeds to the next galaxy in the list.

In Phase II, a larger cylinder is defined around the seed galaxy, with radius $\mathcal{R}_{\mathrm{II}}$ and length $2 \mathcal{L}_{\mathrm{II}}$. We count the number of galaxies in this cylinder that are first or second-order Delaunay neighbors of the seed galaxy, denoting this number by $N_{\text {II }}$. Since the number density of observed galaxies varies with redshift, we

\footnotetext{
17 All VDM cylinder dimensions are comoving distances and are converted to angular and redshift separations by assuming a flat $\Lambda C D M$ cosmology with $\Omega_{M}=0.3$. This cosmology is assumed regardless of the true background cosmology when running on mock catalogs, since it is what we assume when running on the DEEP2 data set, to allow consistency with previous DEEP2 studies, particularly G05. It is straightforward to rescale the cylinder dimensions to different assumed background cosmologies.
} 
correct $N_{\text {II }}$ by the ratio of number density of DEEP 2 galaxies at $z=0.8$ to the local number density at the group redshift. The number density is computed by smoothing the DEEP2 redshift distribution and dividing by the comoving cosmological volume element.

The corrected value, $N_{\mathrm{II}}^{\text {corr }}$, is taken as an initial estimate of the size of the group and is used to scale the final search cylinder in Phase III. The Phase III cylinder is centered on the barycenter of the Phase II galaxies and has radius $\mathcal{R}_{\mathrm{III}}=\max (r \times$ $\left.\left(N_{\text {II }}^{\text {corr }}\right)^{1 / 3}, \mathcal{R}_{\text {min }}\right)$ and length $\mathcal{N}_{\text {III }}=\max \left(\ell \times\left(N_{\text {II }}^{\text {corr }}\right)^{1 / 3}, \mathcal{L}_{\text {min }}\right)$, with $r$ and $\ell$ being the Phase III parameters of the algorithm. All galaxies that fall within the Phase III cylinder are deemed to be members of the group. The algorithm then continues to the next galaxy in the list that has not yet been assigned to a group and repeats the procedure.

The VDM thus has six tuneable parameters (two for the search cylinder in each of the three phases) that must be optimized for a particular survey. These are not fully independent, however. For example, an increase in the size of the Phase II cylinder will increase the typical $N_{\text {II }}$ values and so can be offset by a decrease in the Phase III $r$ and $\ell$ parameters. Furthermore, our group-finding exercise (indeed, any group-finding exercise) can be conceptually subdivided into two steps: group detection, which occurs in Phase I alone, and membership assignment, which occurs in Phases II and III. The parameters that control each of these steps can be tuned more or less independently of one another on the way to determining an optimum set of group-finding parameters.

\subsection{Changes to the G05 Algorithm}

Before we leave the discussion of the VDM algorithm, it is important to make note of a few minor changes that we have made to the VDM algorithm we used in G05. First, we have used a redshift of 0.8 as a reference for correcting $N_{\mathrm{II}}$, since $z=0.8$ is near the peak of the DEEP2 redshift distribution, in contrast to the G05 reference value, $z=0.7$, where the redshift distribution is rising sharply in the main DEEP2 sample.

We also made some important changes to the membershipassignment part of the algorithm. In G05 each group included all galaxies identified in either Phase II or Phase III of the VDM algorithm, regardless of whether or not the Phase III cylinder was larger than the Phase II cylinder. This meant that the Phase II cylinder dimensions had to be kept relatively small so as not to swamp small groups with interloper field galaxies. In testing the VDM on our new mock catalogs, we found that this led to significant fragmentation of larger groups: the Phase II cylinder was too small to accurately estimate their richnesses, so the Phase III cylinder was significantly too small to include all their members.

To some degree, this is unavoidable in a sparsely sampled survey, but we found that we were able to mitigate it by allowing the Phase II cylinder to be quite large, similar in scale to a massive cluster. To gain this advantage while avoiding problems in smaller groups, we decided not to include Phase II galaxies in the final group memberships. That is, we use the Phase II cylinder to get a rough estimate of the number of galaxies in the group by drawing a cylinder that is typically too large and will pick up all the group members and possibly some field galaxies. The scaled Phase III cylinder then refines this estimate and will frequently select only a subset of the Phase II galaxies for the final group. In practice, with a very large Phase II cylinder, the $N_{\text {II }}$ counts often simply include all second-order Delaunay neighbors, with the cylinder simply setting a maximum distance at which such neighbors will be considered. For this reason, we find that varying the Phase II cylinder at relatively large sizes has negligible impact on our results. We thus focus mainly on optimizing the Phase I and III parameters in what follows.

\subsection{Considerations for the EGS}

Because the galaxies targeted in the EGS cover a very broad redshift range with a fixed apparent magnitude limit, the range of galaxy luminosities being probed varies dramatically from low to high redshift, with only very bright $\left(L \gtrsim L^{*}\right)$ galaxies being observed at $z \gtrsim 1$ but extremely faint dwarfs included at low redshift. The presence of these introduces some complications into the group-finding process. The first has to do with the simple definition of a "group." In the main DEEP2 sample, groups are systems containing on the order of a few Milky-Way-sized galaxies at least. At low $z$ in the EGS, by contrast, we will also be capable of detecting systems consisting of a single MilkyWay-sized galaxy and a few dwarfs similar to the Magellanic Clouds. Arguably we should not categorize the latter systems as groups at all.

More importantly, the faint, low- $z$ dwarfs present a challenge for optimizing the VDM group finder. Phases I and II of the VDM algorithm search for galaxies that are connected to a given seed galaxy by one or two links in the Delaunay mesh. Using Delaunay connectedness in this way as a means of detecting groups of bright galaxies rests on the assumption that group members of similar luminosity are likely to be Delaunay neighbors. When the much fainter dwarf galaxies are included, this assumption may break down, since dwarfs are much more numerous than galaxies near $L^{*}$, and so it is possible that a bright galaxy's local Delaunay mesh may be "saturated" by dwarfs, cutting off any links to neighboring bright objects and preventing detection of the larger group. Indeed, in our initial experiments with mock EGS catalogs, we found that it was impossible to achieve satisfactory performance with the VDM group finder at both low and high redshifts simultaneously if the entire EGS galaxy sample was used.

If we choose to focus our group-finding efforts on systems containing multiple bright galaxies, as in the main DEEP2 sample, then fortunately there is a simple way of addressing both of the above issues by limiting Phases I and II of the group finding to bright galaxies only. In particular, when computing the Voronoi partition and Delaunay mesh in the EGS, we can restrict the low-redshift $(z<0.8)$ sample to only those galaxies that are luminous enough that they could have been observed at $z>0.8$. To do this, we follow the procedures used in Gerke et al. (2007), who defined a set of diagonal cuts in the DEEP2 rest-frame (i.e., $k$-corrected as in Willmer et al. 2006) color-magnitude space, which correspond to the DEEP2 $R=24.1$ apparent magnitude limit at different redshifts (see Figure 2 of that paper). If we define such a cut that traces the faint-end limit of DEEP2 galaxies in color-magnitude space at $z=0.8$, we can then select only galaxies brighter than this limit at lower $z$; these are the low-redshift analogs of the main DEEP2 sample. (When performing this selection, we also evolve the cut toward fainter magnitudes at lower redshifts, according to the evolution of $L^{*}$ that was obtained in Faber et al. 2007, namely, a linear evolution of 1.2 mag per unit $z$.)

For EGS group finding, we apply this selection to the $z<0.8$ galaxy population before computing the Voronoi and Delaunay information, and we consider only the selected galaxies in Phases I and II of the algorithm. This means that only systems containing at least two bright galaxies (that would be observable 

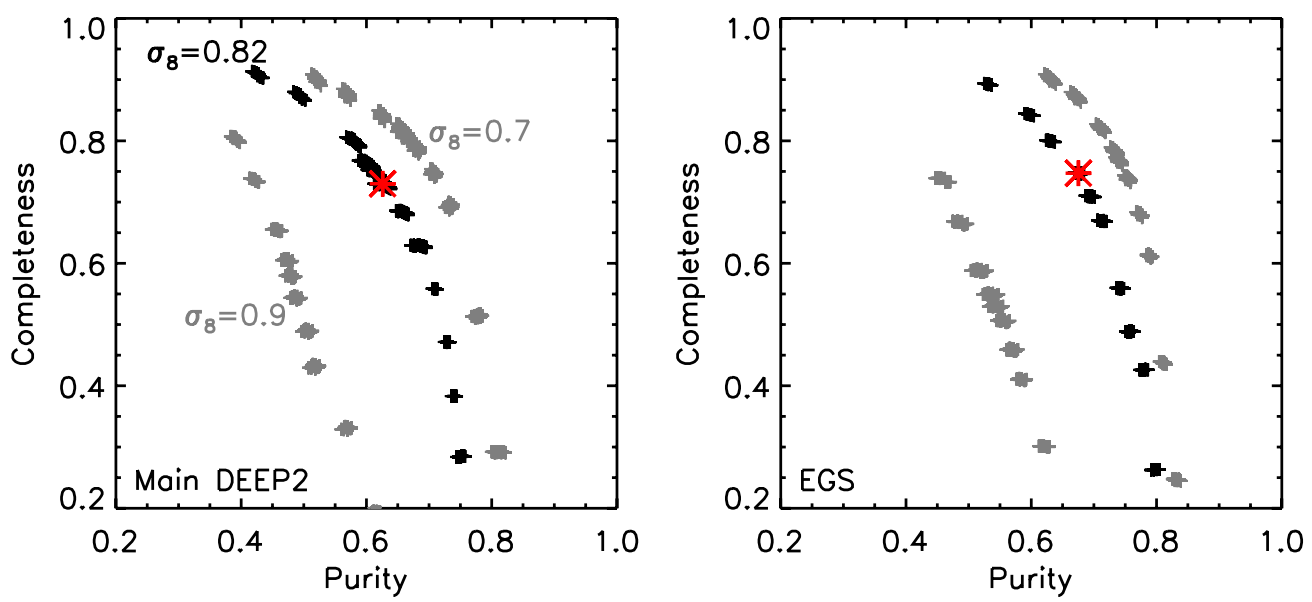

Figure 3. Purity and completeness statistics for group catalogs computed with the VDM group finder over a wide range of the algorithm's parameter space, for each of the three different mock catalogs discussed in Section 2.2. The left panel shows results for mocks selected like the main DEEP2 sample, and the right panel shows results for the EGS mocks. Each point in the plots shows the ${ }^{2} P_{0}$ and ${ }^{2} C_{0}$ statistics (as defined in Section 3.1.4 and computed in aggregate over all mock lightcones) for a particular choice of group-finding parameters in a particular mock catalog. The mock catalogs are identified by the value of $\sigma_{8}$ assumed for each one in the left panel (see Table 1); the relative positions of values for each of the three mocks are the same in the right-hand diagram. The well-known tradeoff between completeness and purity is evident for each mock catalog. There are also substantial differences between the purity and completeness values measured in the different mock catalogs.

(A color version of this figure is available in the online journal.)

at $z \geqslant 0.8$ ) will be counted as groups. In Phase III, however, we consider all galaxies regardless of luminosity, since this final membership-assignment step simply counts all galaxies in the Phase III cylinder, without reference to the Delaunay mesh. This approach to group finding in the EGS has the virtue of ensuring that the groups in the EGS have similar selection, while also counting dwarf members of the groups where they have been observed.

\section{OPTIMIZATION ON DEEP2 MOCK CATALOGS}

The VDM algorithm has six free parameters whose optimal values are not immediately obvious. It is thus very important to test the algorithm on simulated data that reproduce the properties of the real data as accurately as possible. As discussed above in Section 2.2, the mock catalogs developed in B. F. Gerke et al. (in preparation) accurately reproduce a wide array of the observed properties of the DEEP2 catalog, including colordependent selection effects that might disproportionately impact galaxies in groups relative to those in the field. Testing the VDM group finder on these mocks will thus represent a significant improvement over the group-finding effort in G05, which made use of mocks that lacked such color-dependent effects. The current mocks also have been constructed for three different cosmological background models, one of which, for the Bolshoi simulation, is very close to the model that best fits current data.

In practice, we optimize the group-finding parameters by running the VDM group finder repeatedly on the mock DEEP2 observational fields, allowing the group-finding parameters to vary over a wide range in the six-dimensional parameter space, and looking for parameter sets that meet our optimization criteria. Since the Bolshoi simulation cosmology is in the best agreement with present data, we use this simulation to perform the main optimization. However, we repeated this procedure on each of the three different sets of mock catalogs described in Section 2.2 (see Table 1) to test whether and to what degree the optimal parameter set depends on the background cosmology.

For our purposes, the optimal set of group-finding parameters will be the one that most accurately reconstructs the velocity function, as measured using the velocities of the observed galaxies, while also striking the best possible balance between the purity and completeness of the group catalog. It is not immediately obvious that all of these requirements can be met simultaneously within the six-dimensional VDM parameter space. However, the steps in the VDM algorithm divide rather cleanly into a group-detection step (Phase I) and a membershipassignment step (Phases II and III). Since our purity and completeness statistics are mostly a test of group-finding success, whereas velocity dispersion measurements depend on assigning the right galaxies to the right groups, it is reasonable to suppose that the two success criteria may be optimized at least semi-independently. Indeed, experimentation reveals that completeness and purity are only weakly coupled to the shape of the recovered velocity function, at least near the optimum of the purity and completeness values: here, purity and completeness depend mostly on the Phase I parameters of VDM, while the velocity function reconstruction is mainly governed by Phase III.

In the following, then, we optimize purity and completeness first and then consider the velocity function. Additionally, as in G05, we identify a high-purity parameter set, for which the purity of the catalog is nearly maximized, at the expense of completeness. We will use this when constructing the DEEP2 and EGS group catalogs to identify a subset of groups that should be considered higher-confidence detections than the rest.

\subsection{Purity and Completeness}

Figure 3 shows the purity and completeness statistics ${ }^{2} P_{0}$ and ${ }^{2} C_{0}$ that we obtained for widely varying choices of groupfinding parameters in each of the different mock cosmologies. Each data point in the figure represents the completeness and purity values (computed over all mock lightcones for each cosmology) that we obtained for a given set of group-finding parameters and mock cosmology. Results are shown for both the main DEEP2 mocks and the EGS mock catalogs. A diagram like this is a very useful visualization tool for group-finder optimization; it is similar in spirit to Figure 4 of Knobel et al. (2009). The fundamental tradeoff between completeness and purity is readily apparent in the figure: an increase in completeness is always accompanied by a decrease in purity and vice versa. 
For each mock cosmology depicted in the figure, distinct clusters of data points are apparent. In all cases, each individual cluster corresponds to a different value of the Phase I parameter $\mathcal{R}_{\text {min }}$; the impact of varying the other five VDM parameters (rather widely in many cases) is confined to the area of each cluster of points. (The range of $\mathcal{R}_{\text {min }}$ values considered in the figure is $0.1 \mathrm{Mpc} \leqslant \mathcal{R}_{\min } \leqslant 0.5 \mathrm{Mpc}$.) The obvious conclusion is that, for the purposes of optimizing simple group detection, $\mathcal{R}_{\text {min }}$ is by far the most important parameter, with all other parameters having a comparatively negligible effect on detection efficiency. In general, increasing $\mathcal{R}_{\min }$ improves the completeness statistic while degrading the purity (and vice versa). It is apparent from the figure, however, that improvements in either completeness and purity are eventually subject to diminishing returns: at sufficiently low values of $\mathcal{R}_{\text {min }}$, for example, completeness drops rapidly, while purity remains approximately constant. This fact sets a practical range of interest for $\mathcal{R}_{\text {min }}$ (roughly between 0.15 and $0.35 \mathrm{Mpc}$ ), beyond which changes in that parameter only serve to degrade the quality of the catalog. Within this range, the optimal choice of $\mathcal{R}_{\text {min }}$ is debatable, but a reasonable choice (also used by Knobel et al. 2009) is to take the one that gives a result in purity-completeness space that is near the minimum distance from the point $(1,1)$ that is obtained over the full parameter space. We find that this optimum is roughly in the range $0.225 \mathrm{Mpc} \lesssim \mathcal{R}_{\min } \lesssim 0.3 \mathrm{Mpc}$ (the red star denotes $\mathcal{R}_{\text {min }}=0.25$ for the main DEEP2 plot and 0.3 for the EGS plot). Since we will also find below that the velocity function reconstruction depends on $\mathcal{R}_{\text {min }}$, we will allow this parameter to vary slightly in the next step.

The current analysis already allows us to partially identify our high-purity parameter set. Since the purity of the catalog effectively saturates at $\mathcal{R}_{\min }=0.15$, and since purity is not sensitive to any other parameters, we will be able to identify a high-purity subset of the final group catalogs by setting $\mathcal{R}_{\text {min }}$ to 0.15 and holding all other parameters fixed at their optimum values, whatever those turn out to be.

Another notable feature of Figure 3 is the significantly different purity and completeness values we obtained for the different mocks. This implies that our inferred success statistics have a strong dependence on cosmology and particularly on the level of clustering in each dark-matter simulation. This can plausibly be explained as follows. The abundance-matching technique used to construct the mocks requires, by construction, that the luminosity function must match the one that is measured in the DEEP2 data by placing galaxies in halos and subhalos at fixed number density. As discussed in B. F. Gerke et al. (in preparation), as the clustering amplitude $\sigma_{8}$ increases, so does the number density of halos at fixed mass; hence, the abundance-matching algorithm places fainter galaxies in halos (and subhalos) of a given mass. This means in particular that massive halos will contain fewer galaxies above a given luminosity — and thus fewer observable galaxies - as $\sigma_{8}$ increases. This will make these groups more difficult to detect, since their observable galaxy populations will be sparser. For example, the typical halo with two observed members will be more massive in a more clustered cosmology, so its observed members will typically be more widely separated in redshift space. At the same time, increasing $\sigma_{8}$ enhances the clustering of the isolated background galaxies, making them more likely to be erroneously grouped together as false detections. The net effect is that, when the luminosity function is held fixed, increasing the galaxy clustering and halo mass function (e.g., by raising $\sigma_{8}$ and $\Omega_{M}$ ) causes a decrease in both completeness and purity.
This result has important implications for the optimization of group finders generally. Since mock catalogs are usually constructed to reproduce the observed galaxy luminosity function reasonably well, this effect is likely to be generically present in mocks with different background cosmologies and not just in catalogs produced using abundance matching. When assessing group finders, then, it will be very important to construct mocks using simulations whose background cosmology is consistent with the current best-fit cosmological parameters.

Mock catalogs based on semi-analytic galaxy formation models applied to the Millennium Simulation (Springel et al. 2005) have the dual advantages of matching a wide variety of observed properties of the galaxy population and of being easy to obtain and use, so they have been widely used to test and optimize high-redshift group finders. For example, Knobel et al. (2009) used the mock catalogs from Kitzbichler \& White (2007), and Cucciati et al. (2010) constructed mocks using the semianalytic models of De Lucia \& Blaizot (2007) and the lightconeconstruction techniques of Blaizot et al. (2005). However, the Millennium Simulation had a background cosmology with $\sigma_{8}=0.9$, well above the currently preferred value of $\sim 0.8$. Our tests here on mocks with different cosmologies suggest that group finders that were calibrated on the Millennium mocks may have significant inaccuracies in their estimated purity and completeness statistics. (A similar statement can be made about our earlier group-finding efforts in G05 for which this paper should be considered a replacement.) More generally, our results suggest that group catalogs that were calibrated on mocks with disfavored cosmologies should be treated with caution.

When summarizing the success and failure statistics of our DEEP2 group catalog, then, we will use values computed using the Bolshoi mock catalogs only. For the remainder of this section, we will also focus our group-finder optimization efforts on those mocks.

\subsection{The Group Velocity Function}

While maintaining a reasonable balance between completeness and purity of the catalog, we also wish, by tuning the VDM parameters, to produce a catalog of groups that accurately reconstructs the distribution function of observed velocity dispersions for halos in the mock, $d N / d \sigma_{\mathrm{v}}$, at all redshifts of interest. More specifically, we will focus on the high- $\sigma_{\mathrm{v}}$ end of the velocity function, since this region of the distribution is exponentially sensitive to changes in cosmology, and we ultimately wish to use our group catalog for cosmological tests. In G05, we found that VDM could reconstruct the velocity function of the Yan et al. (2004) mocks accurately at $\sigma_{\mathrm{v}} \geqslant 350 \mathrm{~km} \mathrm{~s}^{-1}$; here we will also endeavor to reconstruct the velocity function above a threshold value of $\sigma_{\mathrm{v}}$ that is as low as possible.

Throughout this study, when we discuss the velocity function of groups or halos, we are talking about the measured dispersion $\sigma_{\mathrm{v}}^{\mathrm{gal}}$ of the member galaxies' line-of-sight peculiar velocities (though we will generally drop the superscript for brevity). Since the overwhelming majority of DEEP 2 groups will contain only a few galaxies, we make use of the so-called gapper algorithm to measure $\sigma_{\mathrm{v}}$. The gapper measures velocity dispersion using the gaps between the measured line-of-sight velocities $v_{i}$ in a given group, after the $v_{i}$ have been sorted in ascending order:

$$
\sigma_{G}=\frac{\sqrt{\pi}}{N(N-1)} \sum_{i=1}^{N-1} i(N-i)\left(v_{i+1}-v_{i}\right)
$$


This has been shown to be the most robust of several possible estimators in the limit of small samples (Beers et al. 1990).

In practice, testing the velocity-function reconstruction amounts to comparing the number counts of halos and reconstructed groups in the mock catalogs, in bins of $\sigma_{\mathrm{v}}$ and $z$. In a perfect group finder, these histograms would be exactly equal. To assess the successfulness of the VDM algorithm, we will use a similar approach to the one we used in G05: we compare the fractional error in the recovered histogram to the fractional size of the field-to-field dispersion in the mock histograms. The field-to-field dispersion serves as a proxy for the sample variance (sometimes called cosmic variance) in the group number counts. As a rough rule of thumb, where the error in reconstructing the velocity function is smaller than the sample variance (and also not systematically high or low over a wide range in $\sigma_{\mathrm{v}}$ ), we take the reconstruction to be acceptable, since the measurement error is subdominant to the irreducible uncertainty in the measured velocity function that arises from sampling a finite volume of space. We will endeavor to reduce this error as much as possible, however, focusing mainly on the high-dispersion end of the velocity function, since it is the most sensitive probe of cosmology.

It will be important to be careful in our choice of binning when performing this test. Because the velocity function of groups is quite steep at the high- $\sigma_{\mathrm{v}}$ end, and because the volume of DEEP2 is relatively small, there will be very few groups at large values of $\sigma_{\mathrm{v}}$, especially at high redshift. If we choose a binning in $\sigma_{\mathrm{v}}$ that is too narrow, then most high $\sigma_{\mathrm{v}}$ bins will contain zero or one group, and a slight inaccuracy in the measured velocity dispersion of a given group could lead to very large apparent fractional errors, when in fact the group detection introduces only a minor inaccuracy in the velocity function-one that would have a negligible effect on the inferred cosmology. We would therefore like to use a coarse enough binning to ensure that every bin is expected to contain at least a handful of groups. Since changes in cosmology affect the shape of the velocity function as well as the normalization, though, we would also like to choose a fine enough binning to capture at least some of the shape information.

Somewhat fortuitously, this set of requirements is identical to the one we would use to select a binning for performing cosmological tests with the velocity function: we wish to measure the shape of the velocity function as well as possible given our data set, but standard techniques for constructing likelihood functions over the cosmological parameters assume that the data set contains at least a few objects per bin $(\mathrm{Hu}$ \& Cohn 2006). Hence, for the purposes of testing the VDM velocity-function reconstruction, we will choose a binning that would be appropriate for using the resulting group catalog in cosmological tests, with at least a few groups falling in each bin. Experimenting with the halo population in the mock catalogs, we find that a reasonable choice is to construct even bins in $\log _{10} \sigma_{\mathrm{v}}$, of width 0.1 , with the addition of a single, broad bin covering all values above $\log _{10} \sigma_{\mathrm{v}}=2.75$, with redshift bins of constant width 0.2. Because G05 found that VDM can accurately reconstruct the velocity function above a threshold of $\sigma_{\mathrm{v}}=350 \mathrm{~km} \mathrm{~s}^{-1}$, and lacking any other compelling reason to specify a particular positioning for our bin edges, we choose to arrange our logarithmic bins such that one of the edges falls near the G05 threshold, at $\log _{10} \sigma_{\mathrm{v}}=2.55$.

Within these bins, we compute the fractional difference between the counts of halos and groups and compare it to the fractional sample variance in these counts, as described above.

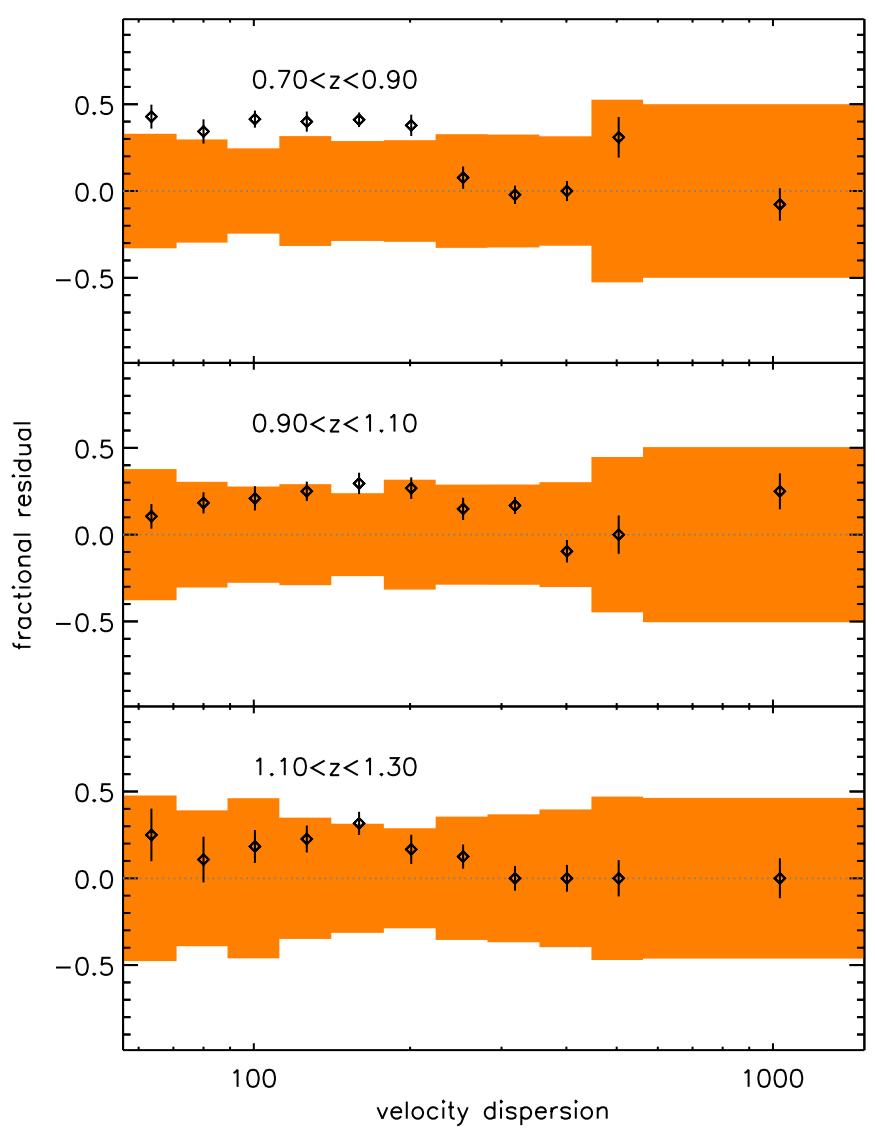

Figure 4. Fractional error in the recovered group velocity function for our optimal set of DEEP2 group-finding parameters (Table 2). Data points show the median fractional difference between the group and halo number counts (defined precisely in the text) in bins of $\sigma_{\mathrm{v}}$ and $z$, for the 40 Bolshoi mock lightcones. Error bars show the standard error computed over the 40 mocks. The shaded regions indicate the fractional scatter in the halo number counts (due to both sample variance and Poisson noise). For $\sigma_{\mathrm{v}} \gtrsim 300 \mathrm{~km} \mathrm{~s}^{-1}$, the fractional errors are consistent with zero and/or are smaller than the sample variance for all redshifts. At lower velocity dispersions, the group abundance is systematically overestimated.

(A color version of this figure is available in the online journal.)

By performing this procedure for group catalogs computed over a wide range in the VDM parameter space, we can search for an optimum set of parameters that minimizes the error in the reconstructed velocity function at high $\sigma_{\mathrm{v}}$. Figure 4 shows the reconstruction error obtained for this optimum set in the Bolshoi mock catalogs. The black points denote the median fractional difference between the binned number counts of groups and halos, $\left(N_{\text {groups }}-N_{\text {halos }}\right) / N_{\text {halos }}$, and the error bars show the standard error in this quantity, with the median ${ }^{18}$ and standard error computed over all 40 Bolshoi mock lightcones. The shaded regions show the fractional sample variance in each bin, as well as indicating the extent of each bin in $\sigma_{\mathrm{v}}$.

To arrive at the parameter set used in the figure, we varied the VDM parameters over a wide range in parameter space (though constraining $\mathcal{R}_{\min }$ to the narrower range of $0.225-0.3$

\footnotetext{
18 We consider the median fractional error in this diagram, rather than the mean, because the former statistic is more resistant to the outlier values we occasionally encounter. Sample variance and shot noise mean that a few of the Bolshoi mock lightcones contain few (in one case zero) halos in one or more of our bins, despite the fact that the typical (i.e., median) lightcone contains at least five halos in each bin. However, the group finder still tends to find at a few groups in all bins, which leads to a very large fractional error for a few lightcones and strongly biases the mean.
} 
Table 2

VDM Group-finding Parameters Used for the Different Sample in This Study

\begin{tabular}{lccc}
\hline \hline Parameter $^{\mathrm{a}}$ & Main & EGS & High Purity \\
\hline $\mathcal{R}_{\min }$ & 0.25 & 0.3 & 0.15 \\
$\mathcal{L}_{\text {min }}$ & 10.0 & $\ldots$ b & $\ldots$ \\
$\mathcal{R}_{\mathrm{II}}$ & 0.8 & $\ldots$ & $\ldots$ \\
$\mathcal{L}_{\text {II }}$ & 8.0 & $\ldots$ & $\ldots$ \\
$r$ & 0.225 & $\ldots$ & $\ldots$ \\
$\ell$ & 10.5 & $\ldots$ & $\ldots$ \\
\hline
\end{tabular}

Notes.

a All values are given in comoving $h^{-1} \mathrm{Mpc}$, assuming a flat $\Lambda \mathrm{CDM}$ cosmology with $\Omega_{M}=0.3$.

b Values not listed are the same as used for the main DEEP2 sample.

identified in the previous section). We used the measured fractional reconstruction errors and their uncertainties (i.e., the data points and error bars in the figure) to construct a statistic similar to $\chi^{2}$ for the bins with $\sigma_{\mathrm{v}}>350 \mathrm{~km} \mathrm{~s}^{-1}$. We then gave detailed consideration to parameter sets near the minimum in this statistic, tuning the parameters by hand to find the parameter set that gives an error in the high- $\sigma_{\mathrm{v}}$ velocity function that is smaller than the sample variance in all bins and is not systematically biased to high or low values. These optimum parameters are listed in Table 2. This set of parameters is also indicated by the red asterisk in Figure 3. It is notable in Figure 4 that this parameter set gives a fractional reconstruction error near zero above a threshold of $\sigma_{\mathrm{v}} \approx 300 \mathrm{~km} \mathrm{~s}^{-1}$, a slight improvement over the value of $350 \mathrm{~km} \mathrm{~s}^{-1}$ we achieved in G05. At lower dispersions, the velocity function is overestimated by the group finder at all redshifts and this bias is larger than the sample variance at low $z$.

While performing the optimization, we found that the reconstructed velocity function was sensitive to the Phase I and Phase III VDM parameters only; the Phase II parameters had no noticeable impact over the range we considered. As discussed in Section 4.2, this is because we have allowed the Phase II cylinder to take very large values, such that we are simply counting all second-order Delaunay neighbors in Phase II in nearly all cases. This suggests that the Phase II cylinder serves little practical purpose in our implementation of the VDM group finder, where we have allowed it to be very large. However, keeping it in place has no negative impact on the recovered catalog, and it has the virtue of ensuring that we never count very distant neighbors in Phase II. On the other hand, if we allowed the Phase II cylinder to take on smaller values the resulting decreases in $\mathcal{N}_{\text {II }}$ could be offset by increases in the Phase III parameters, so that our parameter space for optimization would still be effectively reduced to four parameters, rather than six.

\subsection{The Extended Groth Strip}

Because DEEP2 spectroscopic targets in the EGS were selected differently from those in the main DEEP2 sample, it is reasonable to expect that the optimal parameter set for group finding will also be different. We therefore repeat our parameter optimization procedure separately on the mock lightcones constructed with EGS selection. There are two primary differences between the EGS sample and the main DEEP2 sample. First is the presence of a low- $z$ sample in the EGS, which includes very faint dwarf galaxies. As discussed in Section 4.3, we handle this by excluding from Phase I and II galaxies fainter than those included in the main DEEP2 sample. The second difference is the somewhat denser sampling in the EGS permitted by the use of a four-pass slitmask-tiling algorithm instead of the two-pass approach used in the rest of DEEP2.

This higher sampling rate would naively be expected to make groups easier to detect, and so one might initially suppose that completeness, for example, will rise relative to the rest of DEEP2, at fixed values of the Phase I $\mathcal{R}_{\text {min }}$ parameter. Recall, however, that the Phase I step of VDM looks for first-order Delaunay neighbors, and increasing the density of objects in the galaxy catalog will also shorten the typical linking length in the Delaunay mesh. Since the Phase I cylinder has a long, narrow shape along the line of sight, it is possible that the denser sampling will actually tend to reduce the number of first-order neighbors within the cylinder in favor of nearer neighbors that do not fall along the line of sight. In this case, a slightly larger value for $\mathcal{R}_{\min }$ might be indicated.

In fact, we find the latter situation to be the case: generally speaking, we need a larger value of $\mathcal{R}_{\min }$ to strike a similar balance between purity and completeness to the one we achieved in the main DEEP2 sample. In Figure 3, the red asterisks in both panels sit at approximately the midway point between the maximum attainable purity and completeness values. However, in the EGS (right-hand panel) this point corresponds to $\mathcal{R}_{\min }=0.3$, somewhat larger than the value of 0.25 we used for the main DEEP2 sample. We will therefore use $\mathcal{R}_{\min }=0.3$ when finding groups in the EGS.

One might also initially imagine that the Phase III parameters should be reduced (for fixed Phase II parameters) to account for an expected increase in $\mathcal{N}_{\text {II }}$ values owing to the higher sampling density. However, since $\mathcal{N}_{\text {II }}$ counts only second-order Delaunay neighbors, it is far from clear that its value will be strongly coupled to the sampling density. Indeed, we find that, by increasing $\mathcal{R}_{\min }$ to 0.3 and keeping all other parameters fixed, we can obtain an acceptable reconstruction of the velocity function at high redshifts, $z>0.7$. At lower redshifts, the reconstructed velocity function overestimates the true one slightly; however, the volume probed here is quite small, and we do not plan to use these groups as a cosmological probes. In the interest of constructing a group catalog that is as similar as possible to the main DEEP2 sample, then, we opt to hold all VDM parameters constant in the EGS with the exception of $\mathcal{R}_{\text {min }}$.

\section{THE DEEP2 VDM GROUP CATALOG}

To construct a catalog of DEEP2 galaxy groups, we take all galaxies that yielded good redshifts (DEEP2 quality flag 3 or 4). We then exclude galaxies at $z>1.5$, which are mainly highredshift QSOs and Mg II absorbers. For the main (non-EGS) DEEP2 sample, we also exclude galaxies with $z<0.6$. In the EGS, we exclude galaxies that are too faint for observation at $z>0.8$ in Phases I and II of the VDM procedure, as discussed in Section 5.3. This yields a sample of 22,144 galaxies in the three high-redshift DEEP2 fields and 12,346 galaxies in the EGS. Of these, 3100 high-redshift galaxies (14\% of the sample) and 3070 EGS galaxies (25\% of the sample) are assigned to groups by the VDM group finder. The group fraction in EGS is naturally higher because of the higher sampling density in that field.

\subsection{The Catalogs of Galaxy Groups}

We ran the VDM algorithm on each of these samples using the parameters listed in Table 2. This produces two catalogs for the main sample - one primary catalog and the other high-purity catalog — and two more for the EGS. To identify the high-purity 
Table 3

The DEEP2 VDM Group Catalog

\begin{tabular}{lcccccc}
\hline \hline ID $^{\mathrm{a}}$ & R.A. $^{\mathrm{b}, \mathrm{c}}$ & Decl. ${ }^{\mathrm{b}, \mathrm{c}}$ & $z^{\mathrm{b}}$ & $N$ & $\sigma_{v}{ }^{\mathrm{d}}$ & H.-P. \\
\hline 2052 & 023122.39 & +003519.6 & 0.92 & 6 & 878 & Yes \\
2450 & 022659.36 & +004731.0 & 1.01 & 3 & 873 & Yes \\
2328 & 023033.57 & +003034.2 & 1.04 & 3 & 873 & Yes \\
2151 & 023026.08 & +003804.5 & 0.87 & 4 & 819 & Yes \\
2147 & 022759.14 & +003847.4 & 0.93 & 5 & 813 & No \\
1912 & 233036.04 & +000405.2 & 0.96 & 3 & 930 & No \\
1835 & 233226.54 & +000805.0 & 1.21 & 3 & 894 & Yes \\
1764 & 232941.23 & +001502.8 & 0.83 & 3 & 712 & No \\
2018 & 232550.32 & +002003.0 & 1.00 & 2 & 708 & No \\
1908 & 233315.36 & -000234.4 & 0.89 & 2 & 685 & Yes \\
1416 & 164952.53 & +350553.6 & 1.12 & 3 & 960 & Yes \\
1172 & 165115.36 & +345319.0 & 0.82 & 3 & 813 & Yes \\
1175 & 165309.44 & +350017.5 & 0.86 & 4 & 808 & Yes \\
1186 & 164808.06 & +345608.6 & 0.77 & 3 & 807 & Yes \\
1391 & 165250.72 & +345224.2 & 1.27 & 3 & 667 & Yes \\
998 & 141829.23 & +525040.8 & 0.73 & 3 & 902 & No \\
1078 & 141520.47 & +521929.4 & 0.91 & 4 & 889 & No \\
334 & 142202.89 & +532506.2 & 0.26 & 3 & 853 & Yes \\
857 & 142340.21 & +533159.3 & 0.58 & 3 & 847 & Yes \\
142 & 141530.90 & +521637.4 & 0.46 & 5 & 845 & Yes \\
\hline & & & & & &
\end{tabular}

Notes.

${ }^{a}$ Unique identification number for each group.

b Units of right ascension are hours, minutes, and seconds, and units of declination are degrees, arcminutes, and arcseconds. All values are given for J2000.0 Equinox. The Extended Groth Strip is at $14 \mathrm{hr}$ right ascension.

${ }^{c}$ Median value of all galaxies in the group.

${ }^{\mathrm{d}}$ Given in $\mathrm{km} \mathrm{s}^{-1}$.

e Groups detected with both the standard and the high-purity parameters are indicated as High-Purity.

(This table is available in its entirety in a machine-readable form in the online journal. A portion is shown here for guidance regarding its form and content.)

subset of the main group catalog, we match the primary and high-purity catalogs using the matching techniques described in Section 3.1.3. Groups in the main catalog that are the LAOs of the high-purity groups are then flagged as members of the high-purity catalog.

The main catalog of VDM-identified DEEP2 galaxy groups consists of 1295 groups with $z>0.6$ in the main DEEP2 sample (outside the EGS) and 1165 groups in the redshift range $0<z<1.5$ in the EGS. Of these, 74\% of the main DEEP2 group catalog and $68 \%$ of the EGS group catalog are made up of systems with two observed members (i.e., galaxy pairs). The catalog is presented in Table 3 in which the groups are organized by observational field and sorted by velocity dispersion within each field. Groups that were also detected in the high-purity catalog are identified in the rightmost column of the table. These groups are substantially more likely than the rest to correspond to real dark-matter halos (though they are not guaranteed to do so, just as the other groups in the catalog are not necessarily bogus). Galaxies belonging to the groups are listed in Table 4, with each galaxy's unique DEEP2 identification number listed alongside the ID number of its parent group. These catalogs are also available in electronic format from the DEEP2 data release Web site ${ }^{19}$ they supersede the earlier DEEP2 group catalog presented in G05.

Figure 5 shows the redshift-space distribution of groups in DEEP 2 field 3 (at $23 \mathrm{hr}$ R.A.), which is the DEEP2 field whose spectroscopic observations cover the largest area on the sky

\footnotetext{
19 http://deep.berkeley.edu/dr4
}

Table 4

Galaxies in the DEEP2 VDM Group Catalog and the ID Numbers of Their Host Groups

\begin{tabular}{lc}
\hline \hline Galaxy ID & Group ID \\
\hline 43024982 & 2052 \\
43024870 & 2052 \\
43024803 & 2052 \\
42027302 & 2052 \\
42027156 & 2052 \\
42027036 & 2052 \\
42021576 & 2053 \\
42021537 & 2053 \\
42015342 & 2054 \\
42015248 & 2054 \\
42014311 & 2054 \\
32010356 & 1562 \\
32010351 & 1562 \\
32010253 & 1562 \\
32010001 & 1562 \\
32019550 & 1564 \\
32019541 & 1564 \\
32019539 & 1564 \\
32019392 & 1564 \\
32100597 & 1565 \\
\hline
\end{tabular}

Notes.

${ }^{a}$ Unique galaxy identification number in the DEEP2 catalog.

b Unique identification number for each group, as in Table 3.

(This table is available in its entirety in a machine-readable form in the online journal. A portion is shown here for guidance regarding its form and content.)

(approximately $1 \mathrm{deg}^{2}$ ). Black points in the figure show the positions of field galaxies, while larger circles show groups, with the size of the circle indicating the group richness. Blue circles are members of the high-purity sample; the rest of the groups are indicated in red.

Figure 6 shows distributions of various basic group properties in the EGS and the main DEEP2 catalogs (redshift, richness, and velocity dispersion). To allow for a fairer comparison between the EGS and the main DEEP2 sample, we have also plotted histograms for the subsample of the EGS group catalog at $z \geqslant 0.7$. It is interesting to note that the high-redshift EGS sample contains roughly half as many groups as the rest of DEEP2. This is in spite of the fact that the EGS covers only $20 \%$ of the area covered by the main DEEP 2 sample. The higher observed group abundance in EGS derives partially from the higher sampling rate of galaxies (EGS contains 37\% as many $z>0.7$ galaxies as the rest of DEEP2 combined despite having only a fifth of the area). This means that a larger fraction of low-mass halos will have two observed galaxies in the survey and thus qualify as groups. The higher density of groups also arises partially from the larger value of $\mathcal{R}_{\text {min }}$ we used to detect groups in the EGS. Our purity and completeness values have remained roughly the same as in the rest of DEEP2; since the number of observable halos has increased, this means that the number of false detections must also have increased, which is a result of our larger Phase I cylinder.

\subsection{Success and Failure Statistics of the Catalogs}

We have chosen to optimize our group-finding parameters to accurately reconstruct the velocity function of groups while 

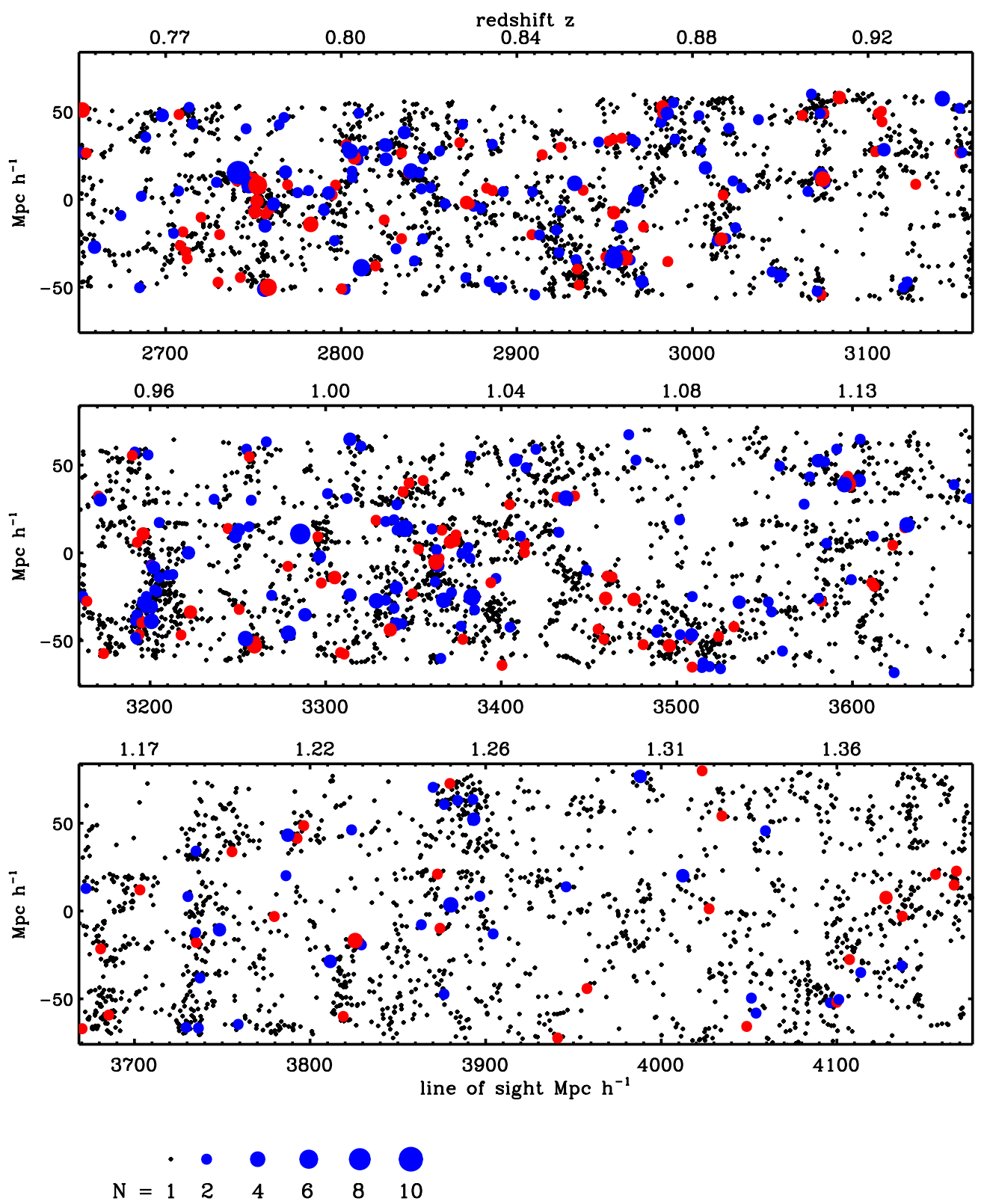

Figure 5. Redshift-space distribution of groups and field galaxies in the largest of the three DEEP2 fields (DEEP2 field 3 , at 23 hr R.A.). Each panel shows a different redshift range, and the three panels taken together represent a contiguous volume covering the redshift range $0.75 \leqslant z \leqslant 1.4$. The vertical axis in each panel shows transverse distance along the right ascension direction on the sky; the plots are projected along the declination direction. Black points show the positions of field galaxies, while red and blue circles show the positions of groups. Blue circles indicate groups that are part of the high-purity subsample and red circles show other groups. The size of each circle indicates the richness of the group. The horizontal empty region about two thirds of the way from the bottom of each panel is caused by a gap in the DEEP2 photometry.

(A color version of this figure is available in the online journal.)

striking a balance between the purity and completeness statistics ${ }^{2} P_{0}$ and ${ }^{2} C_{0}$ for the catalog as a whole. For the catalog we obtain, it will also be useful to compile some of the other success and failure statistics defined in Section 3.1, such as $S_{\text {gal }}$ and $f_{\text {int }}$, both for the catalog as a whole and as a function of redshift and group properties. Many of the success and failure statistics we will discuss in this section are summarized in Table 5.

Figure 7 shows six different definitions of both purity and completeness: ${ }^{w} P_{f}$ and ${ }^{w} C_{f}$ for one-way and two-way matching ( $w=1$ and 2 ) and matching fractions $f=0,0.3$, and 0.5 . Each statistic is shown as computed in bins of redshift, richness, and velocity dispersion. It is notable that, for the catalog as a whole, the different measures of purity and completeness are similar and are all quite flat with redshift. In particular, since the oneway and two-way statistics are quite similar, we can conclude that the fragmentation and overmerging rates are low for the catalog as a whole. However, when we look at the purity and completeness statistics as a function of richness and velocity dispersion, it is clear that group-finding errors become more prevalent for richer and higher $\sigma_{\mathrm{v}}$ groups.

For example, if we consider a stringent $50 \%$ matching fraction, both the completeness and purity of groups with $N>5$ are quite low, even though our usual ${ }^{2} P_{0}$ and ${ }^{2} C_{0}$ statistics are quite high. We also note that the $P_{50}$ and $C_{50}$ curves show an odd-even "sawtooth" pattern in the richness direction. This is a manifestation of the weaknesses of these statistics that we 

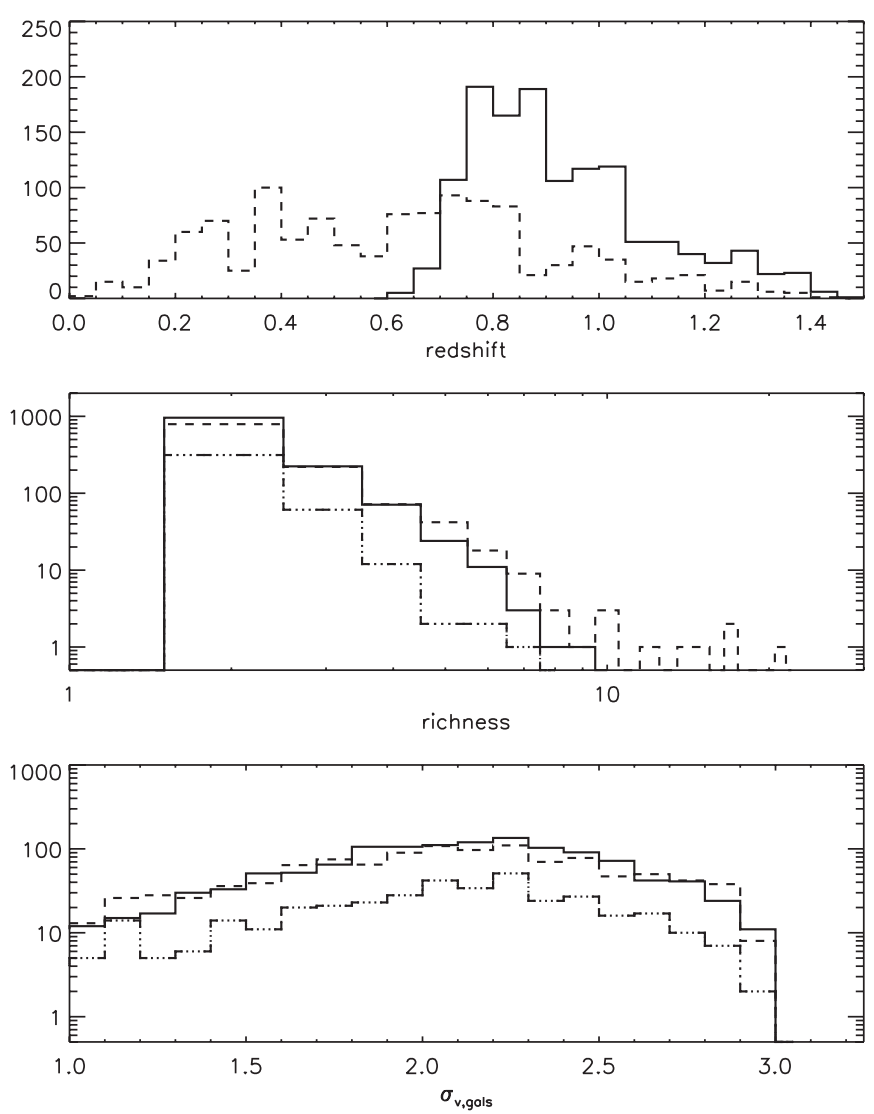

Figure 6. Histograms of various properties of the DEEP2 group catalog. Solid lines show properties of groups in the main sample (excluding the EGS), and dashed lines show groups in the EGS. Dot-dashed lines show EGS groups at $z>0.75$ (for better comparison to the main DEEP2 sample).

discussed in Section 3.1: a halo with $N=5$ needs three detected members to be classified as a successful detection by the ${ }^{2} P_{50}$ standard, but an $N=6$ halo needs four detected members. These plots suggest that, although a large fraction of highrichness halos are successfully detected, many are fragmented and, conversely, although almost none of the high-richness
Table 5

Summary of the Group-finding Success Statistics for the DEEP2 and EGS Group Catalogs

\begin{tabular}{lcccc}
\hline \hline Statistic & Main & Main High Purity & EGS & EGS High Purity \\
\hline${ }^{1} C_{0}$ & 0.75 & 0.49 & 0.79 & 0.45 \\
${ }^{2} C_{0}$ & 0.72 & 0.46 & 0.74 & 0.41 \\
${ }^{1} C_{50}$ & 0.70 & 0.46 & 0.68 & 0.39 \\
${ }^{2} C_{50}$ & 0.68 & 0.44 & 0.66 & 0.38 \\
${ }^{1} P_{0}$ & 0.62 & 0.71 & 0.67 & 0.77 \\
${ }^{2} P_{0}$ & 0.61 & 0.71 & 0.65 & 0.76 \\
${ }^{1} P_{50}$ & 0.54 & 0.62 & 0.55 & 0.63 \\
${ }^{2} P_{50}$ & 0.54 & 0.62 & 0.55 & 0.63 \\
${ }^{\text {gal }}$ & 0.70 & $\ldots$ b & 0.66 & $\ldots$ \\
$f_{\text {int }}$ & 0.46 & $\ldots$ & 0.43 & $\ldots$ \\
$f_{\text {noniso }}$ & 0.04 & $\ldots$ & 0.06 & $\ldots$
\end{tabular}

Notes.

a Uncertainties in the purity and completeness values, as computed from the binomial distribution, are smaller than $1 \%$ and are suppressed here for brevity.

${ }^{\mathrm{b}}$ Galaxy-level statistics are not reported for the high-purity catalogs, since these catalogs simply identify a subset of the groups in the main group catalogs.

groups are false detections, most of them are either overmergers, or have a large interloper fraction, or both.

This situation is unfortunate, but it is also difficult to improve upon. If we make a diagram similar to Figure 3 showing ${ }^{2} P_{50}$ versus ${ }^{2} C_{50}$ for groups with $N \geqslant 4$ only, for example, we can see a similar tradeoff between these two statistics, such that increases in one statistic are generally offset by decreases in the other. We have therefore chosen not to further tune our parameters to improve the high-richness success parameters. Instead, we caution that although high-richness DEEP2 groups can be treated as very high-confidence detections, their memberships may be problematic. It may therefore be advisable, when considering individual high-richness groups, to reconsider their memberships in a non-automated way, e.g., as was done when considering X-ray detected EGS groups in Jeltema et al. (2009).

The success as a function of velocity dispersion is potentially more troubling, especially as regards completeness. The purity is relatively flat as a function of $\sigma_{\mathrm{v}}$, with a small drop around $400 \mathrm{~km} \mathrm{~s}^{-1}$, which likely occurs because groups with a larger Phase III cylinder are likely to have a higher contamination
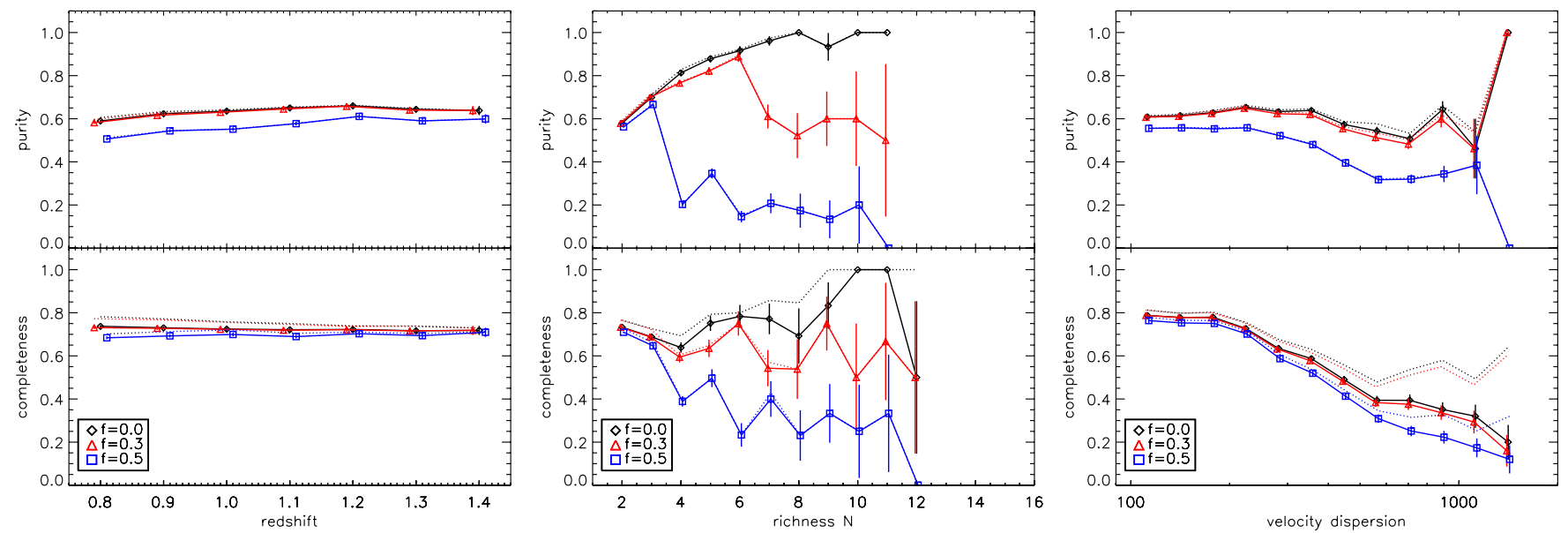

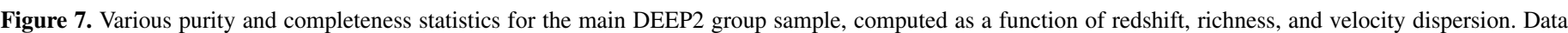

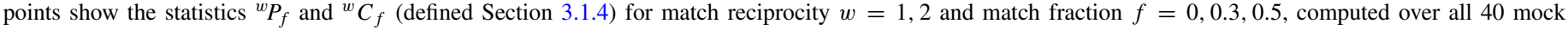

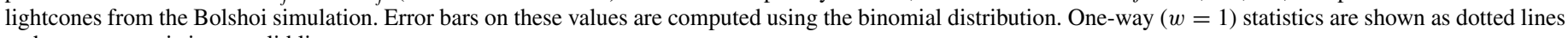
and two-way statistics as solid lines.

(A color version of this figure is available in the online journal.) 
by interlopers. A stronger and more sustained drop in the completeness, ${ }^{20}$ especially the two-way completeness, starts at around $200 \mathrm{~km} \mathrm{~s}^{-1}$. At higher values of $\sigma_{\mathrm{v}}$, the one-way and twoway completeness values are quite different, indicating that the pieces of the high-dispersion halos that VDM detects are being merged with other (presumably lower-dispersion) systems. The drop in completeness can be explained by noting that the halo population is dominated by systems with $N=2$ (i.e., pairs) at all dispersions, and for these systems, $\sigma_{\mathrm{v}}$ is simply proportional to the velocity difference between the members, $\sigma_{\mathrm{v}}=\sqrt{\pi} \Delta v / 2$ (see Equation (1)). Detection in Phase I would require these two galaxies to be first-order Delaunay neighbors, but as they become more widely separated in redshift space, they become increasingly less likely to be linked. Furthermore, the Phase I cylinder half-length has an effective length of $\sim 850 \mathrm{~km} \mathrm{~s}^{-1}$ in velocity at $z \sim 1$, so pairs with $\sigma_{\mathrm{v}} \gtrsim 750 \mathrm{~km} \mathrm{~s}^{-1}$ are completely undetectable in the absence of interlopers. ${ }^{21}$

On the face of it, the rapid drop in completeness with $\sigma_{\mathrm{v}}$ raises concerns about using the VDM catalog to measure the abundance of high-dispersion groups as a cosmological test. The situation may not be as bad as it initially appears, however. As we just explained, the halos that are likeliest to go undetected are systems with two observed galaxies. These will typically be halos at low mass whose measured $\sigma_{\mathrm{v}}$ values have been artificially boosted by small-number statistical effects. Indeed, as shown in the lower right panel of Figure 2, the population of halos above any threshold in $\sigma_{\mathrm{v}}$ includes a very broad range of masses, including many with $M \lesssim 10^{13} M_{\odot}$, owing to the very large scatter in velocity dispersion measured using the observed galaxies in the halos. Such low-mass systems that have been scattered to high $\sigma_{\mathrm{v}}$ are contaminants in the high-mass sample that one would like to construct for use in cosmological tests.

In the Appendix, we develop a theoretical approach to predicting the velocity function of an observed group catalog as a function of cosmological parameters, expanding the Newman et al. (2002) analysis to include observational selection effects and the impacts of contamination from low-mass systems with high measured dispersion values. Although it is essential to include these effects in the theoretical predictions, it is not necessary that the actual contamination in the group catalog arises from exactly the same low-mass halos that have large true velocity dispersions, as long as the contamination in the group catalog is at the same level as expected in the halo population. Since our group catalog accurately reproduces the halo velocity function at high $\sigma_{\mathrm{v}}$ (as shown in Figure 4), it appears that the contamination levels at high dispersion in the halo and group populations are similar. That is, the impurities in the catalog (which are also mostly pairs) account for the undetected pairs at high dispersion. It may thus still be reasonable to use this group catalog for a test like the one proposed in Newman et al. (2002), although it will be important to investigate the incompleteness and contamination of the group catalog very carefully. Such a detailed analysis of the suitability of our group catalog for cosmological tests is beyond the scope of this study though, and

\footnotetext{
${ }^{20}$ It is important to note that this result is quite different from the one shown in Figure 8 of G05, which showed that ${ }^{2} C_{50}$ was flat with $\sigma_{\mathrm{v}}$. As we were testing VDM for this study, we found that figure suffered from a software error which erroneously calculated a flat completeness value. In fact a similar dropoff in completeness was present in the G05 catalog. Since the current study constitutes a replacement for G05, this error should not impact any future studies.

21 An example of such a system is a halo with $\sigma_{\mathrm{v}}^{\mathrm{DM}}=500 \mathrm{~km} \mathrm{~s}^{-1}$ and two observed members with typical velocities that are oriented in opposite directions along the line of sight.
}

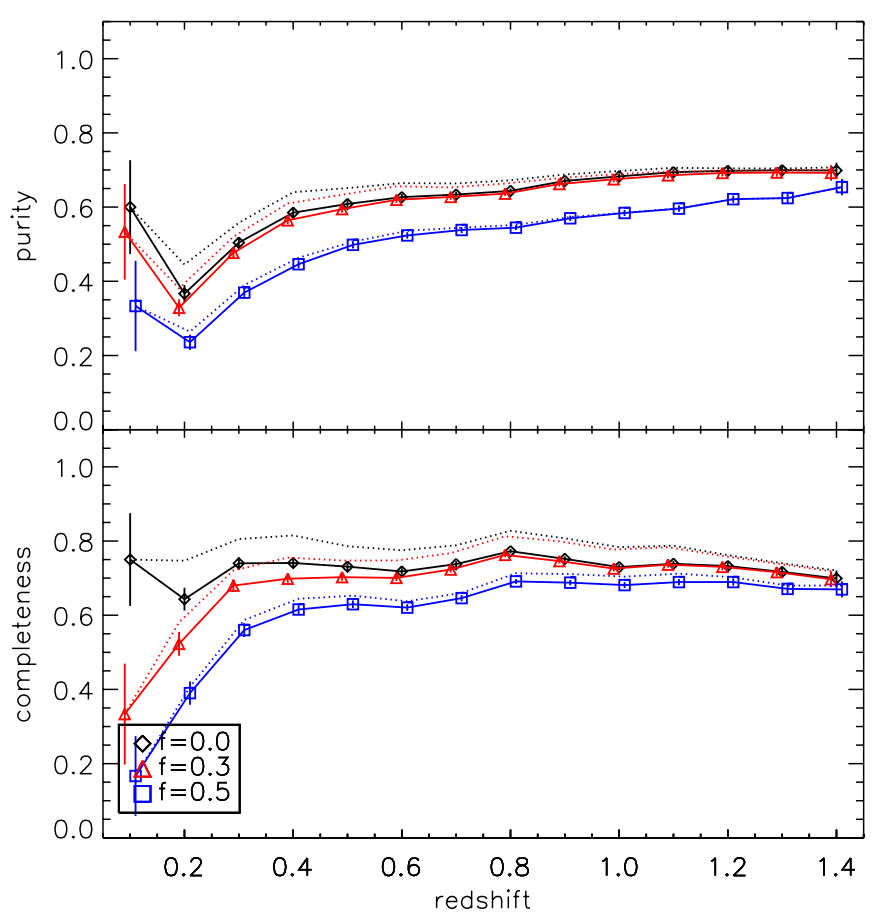

Figure 8. Similar to the left-hand panel of Figure 7, but for the EGS mock catalogs.

(A color version of this figure is available in the online journal.)

apart from a brief discussion in the Appendix, we defer it to future work.

In the case of the EGS, we find similar (though noisier) trends of purity and completeness with $N$ and $\sigma_{\mathrm{v}}$. As shown in Figure 8, however, the purity and completeness statistics are roughly constant at high redshift, but they degrade somewhat at the redshifts below those probed in the rest of DEEP2. The likely reason for this has to do with the effective sampling rate of bright group members in the low-redshift EGS. As we discussed in Section 4.3, when we perform VDM Phases I and II in this regime, we consider only galaxies bright enough that they could have been observed at $z \geqslant 0.8$. The EGS target-selection downweights low-redshift galaxies so that the redshift distribution of targets is roughly constant, and since the low-redshift pool of targets also includes faint galaxies that might be observed in lieu of bright ones, the sampling rate of bright galaxies is sharply reduced at low redshift relative to what can be attained at high redshift. A more sparsely sampled galaxy population is certain to result in a lower-quality group catalog, since there are fewer observable halos overall, and their members are more widely separated. Thus, although we have attempted to select groups as uniformly as possible, there are limits to how well this can be accomplished. For this reason, we advise particular caution when comparing the low-redshift group catalog in the EGS to the rest of DEEP2.

In addition to identifying individual groups, the group catalog also partitions the galaxy catalog into a set of group members and a set of field galaxies. Since galaxy groups and clusters are an interesting environment for studying galaxy formation and evolution, it will be worthwhile to consider the success and failure statistics for the galaxy population that we defined in Section 3.1. These statistics, computed for the catalogs as a whole, are summarized in Table 5. As shown in Figure 9, these values are nearly constant with redshift at high $z$; however, there is a significant worsening of the galaxy success statistics at low 

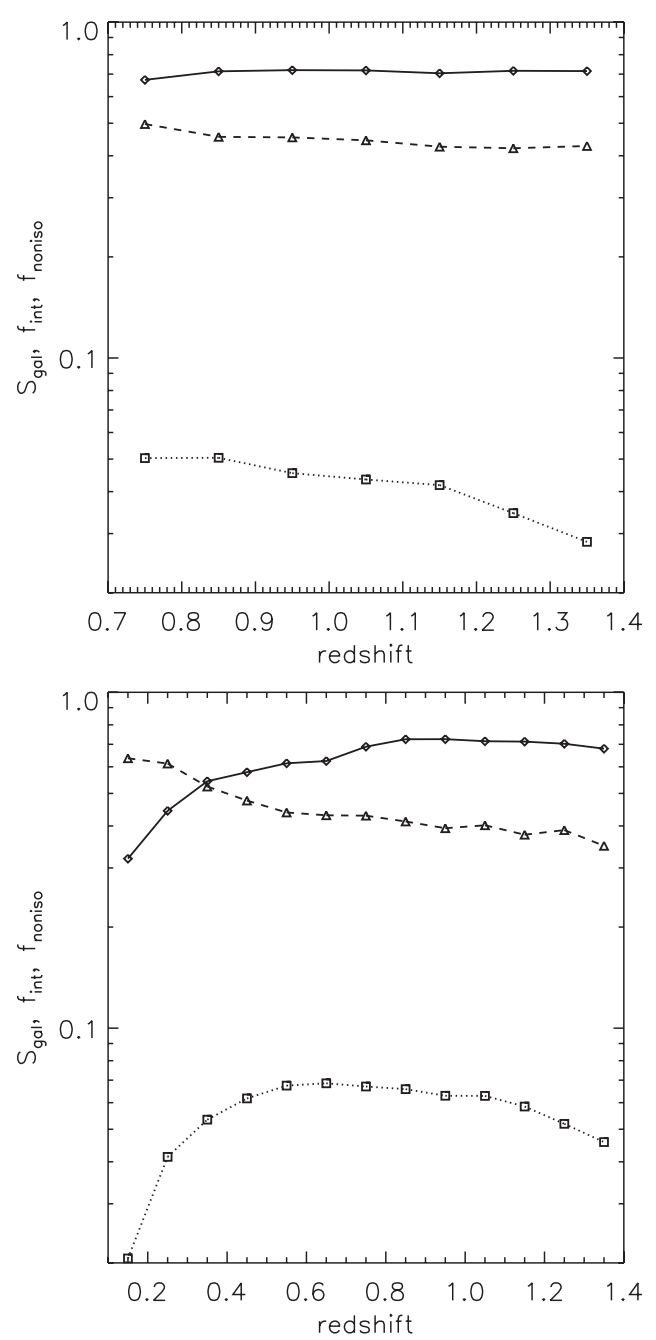

Figure 9. Galaxy-level success and failure statistics (defined in Section 3.1.2 for the main DEEP2 sample (top) and EGS (bottom)), as a function of redshift. The solid lines show the galaxy success rate, $S_{\text {gal }}$; dashed lines show the interloper fraction, $f_{\text {int }}$ and dotted lines show the non-isolated fraction, $f_{\text {noniso }}$.

redshift in the EGS. The increase in interloper fraction at low redshift is likely caused by the presence of faint isolated galaxies that happen by chance to fall in the Phase III cylinder of some group (the Phase III cylinder can be many tens of Mpc long in comoving line-of-sight distance to account for the finger-ofgod effect.) The reduction in $S_{\text {gal }}$ likely derives from the lower sampling rate in the low- $z$ EGS that we discussed above.

Finally, we note that within the halo model for galaxy formation, it is natural to divide the galaxy population into central galaxies, which are located at the centers of distinct dark-matter halos, and satellite galaxies, which are located in subhalos. Considering the properties and evolution of centrals and satellites separately has recently been a popular and fruitful approach to galaxy evolution studies. Ideally, one would hope that the population of field galaxies identified by a group finder would constitute a relatively pure sample of central galaxies that could be studied separately from the satellite population. In tests on the DEEP2 mock catalogs, we find that the fraction of field galaxies that are actually satellites is $11 \%$, which is a mild improvement over the $16 \%$ satellite fraction in the overall population of galaxies in the mock. (These numbers are $12 \%$ and $18 \%$, respectively, in the EGS mocks.) The satellite fraction can be higher than $f_{\text {noniso }}$ since some truly isolated galaxies in DEEP2 will be satellites if their central galaxies were not scheduled for DEIMOS spectroscopy or did not yield good redshifts (this also means that even a perfect group finder would not yield a satellite fraction of zero for the field galaxies).

\subsection{Comparison to Other High-redshift Spectroscopic Group Catalogs}

There are two other spectroscopic galaxy surveys in the literature that bear a particular resemblance to DEEP2 in targeting galaxies at redshift of order unity with sample sizes on the order of tens of thousands of galaxies. These are the VVDS (Le Fèvre et al. 2005) and zCOSMOS (Lilly et al. 2009) samples. Group catalogs have been computed for both of these samples using the VDM group finder by Cucciati et al. (2010, hereafter C10) and Knobel et al. (2009, hereafter K09), respectively. (The latter authors also computed a group catalog using an FoF algorithm and used information from both algorithms to construct a final group catalog for zCOSMOS.) Since the samples and methods used in those studies are broadly similar to ours here, it will be useful to compare the DEEP2 group catalog with the VVDS and zCOSMOS results.

The DEEP2 group catalog presented here is larger than the VVDS and zCOSMOS catalogs, and it probes higher typical redshifts. The DEEP2 catalog contains 3016 groups in total, compared with 318 in VVDS and 1473 in zCOSMOS. The latter two catalogs lie primarily in the range $0.2<z<1.0$, with only a few systems at higher $z$, whereas the DEEP2 catalog focuses on the range $0.7<z<1.4$.

There are important differences among the reported success and failure statistics of the various catalogs. As we do, C10 find that they can accurately reconstruct the velocity function of groups for $\sigma_{\mathrm{v}} \gtrsim 350 \mathrm{~km} \mathrm{~s}^{-1}$. K09 do not consider the velocity function, but their VDM catalog significantly overestimates the group abundance at all richnesses, while their combined FoF and VDM catalog accurately reconstructs the abundance. $\mathrm{C} 10$ and K09 both report purity and completeness values using the one-way and two-way $P_{50}$ and $C_{50}$ statistics, so we can compare our catalog with theirs on those terms. We will focus on two-way statistics, since these are the most stringent test of group-catalog quality and since the one-way and two-way statistics for our catalog are quite similar. Table 5 summarizes various purity and completeness statistics for the DEEP2 catalog. In terms of overall purity and completeness, this catalog, with $\left({ }^{2} P_{50},{ }^{2} C_{50}\right)=(0.54,0.68)$, is intermediate between the zCOSMOS $(0.72,0.78)$ values and the VVDS values of $(0.49,0.53)$. This is not unexpected, since the DEEP2 sampling rate is intermediate between the other two surveys, and sampling density is the most important factor governing group-finding success in redshift space.

When we consider the completeness and purity for highrichness groups, however, there are some rather striking differences between the DEEP2 catalog and the other two. As shown in Figure 7, the DEEP2 $P_{50}$ and $C_{50}$ statistics are rather low for groups and halos with more than three members. By contrast, both $\mathrm{C} 10$ and $\mathrm{K} 09$ report purity and completeness statistics that are nearly flat with richness. As we discussed above, we have not been able to find a parameter set that increases the high-richness purity or completeness without causing a decline in the other parameter. We have run VDM on the DEEP2 (Bolshoi) mock catalogs using the optimal parameter sets determined in $\mathrm{C} 10$ and K09 and have found that in both cases the high-richness completeness is substantially improved, but this is at the expense of the purity. In any event, we note that the ${ }^{2} P_{0}$ and ${ }^{2} C_{0}$ statistics are quite high for our high-richness halos and groups, so it is not 
correct to say that they are mostly failures. Instead, the membership assignment appears to be somewhat problematic for these systems.

There are a few possible explanations for the differences between our high-richness statistics and the others. In the case of $\mathrm{K} 09$, it may be possible to partially or fully account for the difference by the fact that zCOSMOS has a higher sampling density than DEEP2. However, the difference is quite puzzling in the case of $\mathrm{C} 10$, since the VVDS sampling rate is substantially lower than DEEP2's. Another difference that distinguishes both the $\mathrm{C} 10$ and the $\mathrm{K} 09$ catalogs from ours is their redshift range covered and the resulting luminosity range probed. The main DEEP2 sample at $z>0.75$ only probes galaxies with $L \gtrsim L^{*}$ (Willmer et al. 2006), so a DEEP2 group with five members, say, has five bright members and thus is quite massive. With similar flux limits to DEEP2, but lower redshift ranges, VVDS and zCOSMOS will probe faint dwarf galaxies at low redshift, and only brighter galaxies at high redshift, so that systems with five members will be much less massive at $z=0.1$ than at $z=0.9$. The presence of the fainter galaxies in lower-mass systems means that the typical effective sampling density of groups will be higher in VVDS and zCOSMOS than it is in DEEP2 (recall that we exclude faint galaxies from the groupdetection step at low redshift in the EGS). This may make the detection of high-richness groups easier in the lower $z$ samples.

A final potential explanation for the differences in reported purity and completeness statistics is the differences between the mock catalogs used to estimate these values. As we showed in Section 5.1, small differences in the background cosmology of a mock catalog can lead to significant differences in the measured purity and completeness statistics for the VDM group finder. $\mathrm{C} 10$ and K09 both utilized mock catalogs based on the Millennium Simulation, whose cosmological parameters (particularly the value $\sigma_{8}=0.9$ ) lie well outside the range preferred by current data. In the case of our mock catalogs, a higher value of $\sigma_{8}$ leads to depressed purity and completeness statistics, but since the Millennium mocks used a different prescription for adding galaxies than we do, it is difficult to intuit the effect that the background cosmology will have on the inferred success and failure statistics. Nevertheless, since properties of the mock galaxies (e.g., the luminosity function) match observations, while the halo population is not consistent with the favored cosmology, the halo occupation statistics of galaxies are likely to be inaccurate. One must therefore interpret purity and completeness statistics computed from these mocks with some caution.

In this study, we have made a substantial effort to use mocks (the Bolshoi mocks) whose galaxy population and background cosmology are as consistent as possible with a wide range of observations. We have also taken pains to ensure that we are striking the best possible balance between completeness and purity given the stated goals of our group-finding efforts. Although the $\mathrm{C} 10$ and K09 catalogs nominally appear to have better purity and completeness than ours (at high richness and under very stringent definitions of purity and completeness), given the differences between our mock catalogs and theirs, it is nevertheless difficult to say whether this reflects a true difference among the data sets or an insufficiently realistic set of mocks in the earlier studies.

\section{CONCLUSION}

We have constructed and released to the public a catalog of galaxy groups detected in redshift space from the spectroscopic galaxy sample in the DEEP2 Galaxy Redshift Survey, including the EGS. Groups are detected using the VDM group finder (Marinoni et al. 2002), as optimized on new, highly realistic mock galaxy catalogs, using similar techniques to the ones we developed in G05 for early DEEP2 data, using a previous generation of DEEP2 mocks. The catalogs developed here replace those presented in G05. Because galaxies in the EGS region are selected with a different algorithm than used in the rest of DEEP2, we perform a separate optimization of the group finder on mock catalogs specially constructed to resemble the EGS. We also chose to exclude faint, low-redshift galaxies from the first steps of group finding in the EGS, to ensure that the selection of the EGS group catalog is approximately uniform in redshift and similar to the rest of DEEP2.

In the course of performing the optimizations, we developed a generalized definition of the purity and completeness of the group catalog, which avoids some problems that arise under the definitions we used in G05. We argue in particular that the G05 definition is overly stringent and, by construction, gives results that are strongly (and spuriously) richness dependent for low-richness systems. Using our more general purity and completeness measures, we find that our optimized group catalog is $72 \%$ complete and $61 \%$ pure for the main DEEP2 sample, and $74 \%$ complete and $67 \%$ pure for the EGS. We also construct a group catalog using parameters chosen to maximize purity (at the expense of completeness), and we make note of these systems in our main catalog. The high-purity subsample is $71 \%$ pure (76\% in the EGS). We also estimate our success in partitioning the galaxy catalog into group and field subsamples. We find that our group finder correctly identifies $70 \%$ of true group galaxies, with $46 \%$ of the identified group galaxies being interlopers from the field, while $4 \%$ of identified field galaxies are actually members of groups (these numbers are 66\%, $43 \%$ and $6 \%$, respectively, in the EGS). A more complete listing of group-finding success and failure statistics, including different definitions of the completeness and purity, is given in Table 5.

To optimize the group-finding parameters, we made use of mock catalogs constructed using three different $N$-body simulations, with varying values of the cosmological parameters $\Omega_{M}$ and $\sigma_{8}$. The purity and completeness values we estimate from these catalogs vary quite strongly with the assumed cosmology. This underscores the importance of optimizing the group finder on mocks that are consistent with as broad a range of data as possible, including both cosmological information and the properties of the galaxy population. We have taken care in this work to report statistics from mock catalogs that match the galaxy data and are consistent with all current cosmological constraints. We note that earlier high-redshift group-finding efforts (e.g., G05; K09; C10) have been optimized on mocks with now-disfavored cosmologies, so their reported purity and completeness statistics should be treated with caution.

As we did in G05, we find that the optimized VDM groupfinding algorithm can reconstruct the distribution of groups in velocity dispersion and redshift for the main DEEP2 sample, above a threshold in dispersion of $\sigma_{\mathrm{v}} \sim 300 \mathrm{~km} \mathrm{~s}^{-1}$, to an accuracy better than the sample variance in this distribution. Moreover, our optimal parameter set can achieve this level of accuracy for all three mock cosmologies considered here, in spite of the different purity and completeness statistics obtained for the different mocks. As shown in Newman et al. (2002), the velocity function of groups can be used as a probe of cosmological parameters; the fact that VDM can accurately reconstruct this distribution for a range of cosmologies makes 
the DEEP2 group catalog presented here a promising data set for this test, although a more detailed accounting for sources of incompleteness and contamination in the group catalog will be important in any such analysis.

We thank C. Marinoni for making the VDM group-finding algorithm available for our use. This work was supported in part by the NSF grants AST-0507428, AST-0507483, AST-0071048, AST-0071198, AST-0808133, and AST-0806732. B.F.G. was supported by the U.S. Department of Energy under contract number DE-AC02-76SF00515. M.C.C. received support from NASA through the Hubble Fellowship grant HF-51269.01-A awarded by the Space Telescope Science Institute, which is operated by the Association of Universities for Research in Astronomy, Inc., for NASA, under the contract NAS 5-26555; and from the Southern California Center for Galaxy Evolution, a multi-campus research program funded by the University of California Office of Research. The data presented herein were obtained at the W. M. Keck Observatory, which is operated as a scientific partnership among the California Institute of Technology, the University of California, and the National Aeronautics and Space Administration. The Observatory was made possible by the generous financial support of the W. M. Keck Foundation. The DEIMOS spectrograph was funded by a grant from CARA (Keck Observatory), an NSF Facilities and Infrastructure grant (AST92-2540), the Center for Particle Astrophysics, and by gifts from Sun Microsystems and the Quantum Corporation. The DEEP2 Redshift Survey has been made possible through the dedicated efforts of the DEIMOS staff at UC Santa Cruz who built the instrument and the Keck Observatory staff who have supported it on the telescope. Finally, the authors wish to recognize and acknowledge the very significant cultural role and reverence that the summit of Mauna Kea has always had within the indigenous Hawaiian community. We are most fortunate to have the opportunity to conduct observations from this mountain.

\section{APPENDIX}

\section{ANALYTICAL TECHNIQUE FOR PREDICTING THE VELOCITY FUNCTION OF GROUPS}

One of the primary stated purposes of this study is to create a catalog of massive systems that can be used to constrain cosmological parameters by measuring the observed distribution of groups in redshift and velocity dispersion. As a service to future users of the catalog, we outline here a method for computing this distribution as a function of cosmological parameters. All of the calculations outlined below can be straightforwardly performed to include redshift dependence; we do not explicitly include this for succinctness.

The goal is to constrain the mass function of dark-matter halos, whose dependence on cosmology is well understood theoretically, using the measured velocity dispersion of groups as a proxy for halo mass. Doing this requires an analytical technique for converting from the halo mass function to the velocity function, i.e., the distribution of galaxy groups as a function of their observed velocity dispersion $\sigma_{\mathrm{v}}$. When forecasting the constraints that might be possible from DEEP2, Newman et al. (2002) developed a method for converting from the mass function to the halo velocity function, as would be measured using the dark-matter velocity dispersion $\sigma_{\mathrm{v}}^{\mathrm{DM}}$, but that analysis did not account for the substantial additional scatter in the $M-\sigma_{\mathrm{v}}$ relation arising from observational selection effects and the limited number of galaxies used to calculate each group's $\sigma_{\mathrm{v}}$ (as shown in Figure 2).

In any case, the first step is to compute the halo mass function $d n / d M$ and convert it to the halo velocity function $d n / d \sigma_{\mathrm{v}}^{\mathrm{DM}}$. The theoretical halo mass function has a relatively simple dependence on cosmological parameters and has been calibrated against $N$-body simulations to an accuracy better than required for DEEP2 comparisons (e.g., Tinker et al. 2008 and references therein). The relation between halo mass $M$ and $\sigma_{\mathrm{v}}^{\mathrm{DM}}$ is also well calibrated from $N$-body simulations (Evrard et al. 2008 ), so we can use this relation to convert from $d n / d M$ to $d n / d \sigma_{\mathrm{v}}^{\mathrm{DM}}$ directly. ${ }^{22}$ Then the observed velocity function takes the form of a convolution to account for observational scatter:

$$
d n / d \sigma_{\mathrm{v}}^{\mathrm{gal}}=\int_{0}^{\infty} d n / d \sigma_{\mathrm{v}}^{\mathrm{DM}} P\left(\sigma_{\mathrm{v}}^{\mathrm{gal}} \mid \sigma_{\mathrm{v}}^{\mathrm{DM}}\right) d \sigma_{\mathrm{v}}^{\mathrm{DM}}
$$

where $\sigma_{\mathrm{v}}^{\mathrm{gal}}$ is the velocity dispersion measured using the observed galaxies in the group.

The remaining task is then to compute the conditional probability distribution function (PDF) in Equation (A1). Provided that the number of galaxies $N$ per halo is known, and assuming that these galaxies randomly sample the dark-matter velocity field (i.e., that there is no velocity bias) and that the underlying velocity distribution is normal, the problem reduces to computing the distribution of measured dispersions obtained by sampling a Gaussian $N$ times. The form of this distribution depends on the estimator we use to compute the measured dispersion. For the usual standard deviation estimator, it can be computed analytically (Kenney 1951):

$$
P_{\mathrm{sd}}\left(\sigma_{\mathrm{v}}^{\mathrm{gal}} \mid \sigma_{\mathrm{v}}^{\mathrm{DM}}, N\right) \propto \frac{1}{\sigma_{\mathrm{v}}^{\mathrm{DM}}}\left(\frac{\sigma_{\mathrm{v}}^{\mathrm{gal}}}{\sigma_{\mathrm{v}}^{\mathrm{DM}}}\right)^{N-2} \exp \left[-\frac{N}{2}\left(\frac{\sigma_{\mathrm{v}}^{\mathrm{gal}}}{\sigma_{\mathrm{v}}^{\mathrm{DM}}}\right)^{2}\right],
$$

with the normalization being set by the condition $\int_{0}^{\infty} P d \sigma_{\mathrm{v}}^{\mathrm{gal}}=1$.

Our preferred estimator, however, is the gapper, Equation (1). The sorting of the data points required by that estimator makes analytical computation of the desired conditional PDF intractable for $N>2$. However, for the $N=2$ case, we can derive a closed form, via a calculation very similar to the one that produced Equation (A2):

$$
P_{G}\left(\sigma_{\mathrm{v}}^{\mathrm{gal}} \mid \sigma_{\mathrm{v}}^{\mathrm{DM}}, N=2\right) \propto \frac{1}{\sigma_{\mathrm{v}}^{\mathrm{DM}}} \exp \left[-\frac{1}{\pi}\left(\frac{\sigma_{\mathrm{v}}^{\mathrm{gal}}}{\sigma_{\mathrm{v}}^{\mathrm{DM}}}\right)^{2}\right] .
$$

The notable similarity between the $N=2$ cases of $P_{G}$ and $P_{\text {sd }}$ suggests a possible approximate form for $P_{G}$ in the case of general $N$,

$$
\begin{gathered}
P_{G}\left(\sigma_{\mathrm{v}}^{\mathrm{gal}} \mid \sigma_{\mathrm{v}}^{\mathrm{DM}}, N\right) \propto \frac{1}{\sigma_{\mathrm{v}}^{\mathrm{DM}}}\left(\frac{\sigma_{\mathrm{v}}^{\mathrm{gal}}}{\sigma_{\mathrm{v}}^{\mathrm{DM}}}\right)^{N-2} \\
\times \exp \left[-\frac{\alpha(N)}{2}\left(\frac{\sigma_{\mathrm{v}}^{\mathrm{gal}}}{\sigma_{\mathrm{v}}^{\mathrm{DM}}}\right)^{2}\right]
\end{gathered}
$$

\footnotetext{
22 In principle there is some scatter about the $M-\sigma_{\mathrm{v}}^{\mathrm{DM}}$ relation, so the conversion should actually take the form of a convolution of the mass function with the distribution of $\sigma_{\mathrm{y}}^{\mathrm{DM}}$ at fixed $M$, but in practice this scatter is much smaller than the observationally induced scatter we discuss below, so it can be neglected in favor of a simple change of variables.
} 
where $\alpha(N)$ is a free parameter that needs to be determined by numerical calculations. Monte Carlo experiments indicate that the one-parameter fitting formula, Equation (A4), is a remarkably good model for the $\sigma_{\mathrm{v}}^{\mathrm{gal}}$ PDF. By fitting this function to Monte Carlo simulated distributions over the range $2 \leqslant N \leqslant 25$, we find that the parameter $\alpha(N)$ is well described by

$$
\alpha(N)=N-2\left(1-\frac{1}{\pi}\right)\left(1+\frac{1-e^{-N / 2+1}}{10}\right)
$$

Armed with the above formula for $P_{G}\left(\sigma_{\mathrm{v}}^{\mathrm{gal}} \mid \sigma_{\mathrm{v}}^{\mathrm{DM}}, N\right)$, we can write down a form for the desired conditional PDF:

$$
P\left(\sigma_{\mathrm{v}}^{\mathrm{gal}} \mid \sigma_{\mathrm{v}}^{\mathrm{DM}}\right)=\sum_{N_{\mathrm{obs}}=2}^{\infty}\left[P\left(N_{\mathrm{obs}} \mid \sigma_{\mathrm{v}}^{\mathrm{DM}}\right) P\left(\sigma_{\mathrm{v}}^{\mathrm{gal}} \mid \sigma_{\mathrm{v}}^{\mathrm{DM}}, N_{\mathrm{obs}}\right)\right]
$$

where $N_{\text {obs }}$ is the number of observed galaxies in a particular halo. The lower limit of the sum obtains because systems with $N_{\text {obs }}<2$ do not qualify as groups; we note that this sum thus also implicitly encodes the halo selection function in full detail, provided that we have an accurate form for $P\left(N_{\mathrm{obs}} \mid \sigma_{\mathrm{v}}^{\mathrm{DM}}\right)$.

What remains, then, is to determine $P\left(N_{\text {obs }} \mid \sigma_{\mathrm{v}}^{\mathrm{DM}}\right)$. This can be done in principle using sufficiently realistic mock catalogs to measure the desired PDF directly. Since this is likely to be noisy, however, it may be preferable to use the techniques of the halo model: one can simply measure the observed HOD $\bar{N}_{\text {obs }}(M)$ and assume a simple well-motivated model for the scatter about this mean, such as the model of Zheng et al. (2005), who use Poisson scatter for satellites and a nearestinteger distribution for centrals. We note that, since this is the HOD of observed galaxies, including all spectroscopic selection effects, the form of the HOD will be different from the one that is usually assumed - for example, the mean number of centrals will always be less than unity-but the scatter model should still be applicable.

Thus, given sufficiently realistic mock catalogs, it is possible to predict the observed group velocity function analytically. The question of what constitutes sufficient realism in the mocks warrants brief discussion, though. Since we are using the mocks to compute the observed HOD, we would like them to accurately (1) reflect the true total HOD $\bar{N}(M)$ in the universe and (2) include all selection effects relevant to the data set being used. The first requirement is equivalent to demanding that the mocks reproduce the observed two-point correlation function of galaxies, and the second necessitates careful modeling of galaxy properties to match the data. As discussed above and in B. F. Gerke et al. (in preparation), the mock catalogs we use here meet the latter requirement but not the former. However, as also discussed in B. F. Gerke et al. (in preparation), the improvements to the mock catalogs that are likely to improve the clustering agreement are also likely only to impact the lowmass portion of the HOD, where $\vec{N} \lesssim 1$. Since the above formalism only relies on objects with $N_{\text {obs }}>2$, there will be little contribution from this inaccurate portion of the HOD. Thus, it is possible that the mocks we used in this study are sufficiently accurate to carry out the modeling described here; however, this assumption would need to be tested in some detail before robust cosmological constraints could be derived from the velocity function of DEEP2 groups.

A simple way to test this within the confines of the present study is to compare the abundance of DEEP2 groups in bins

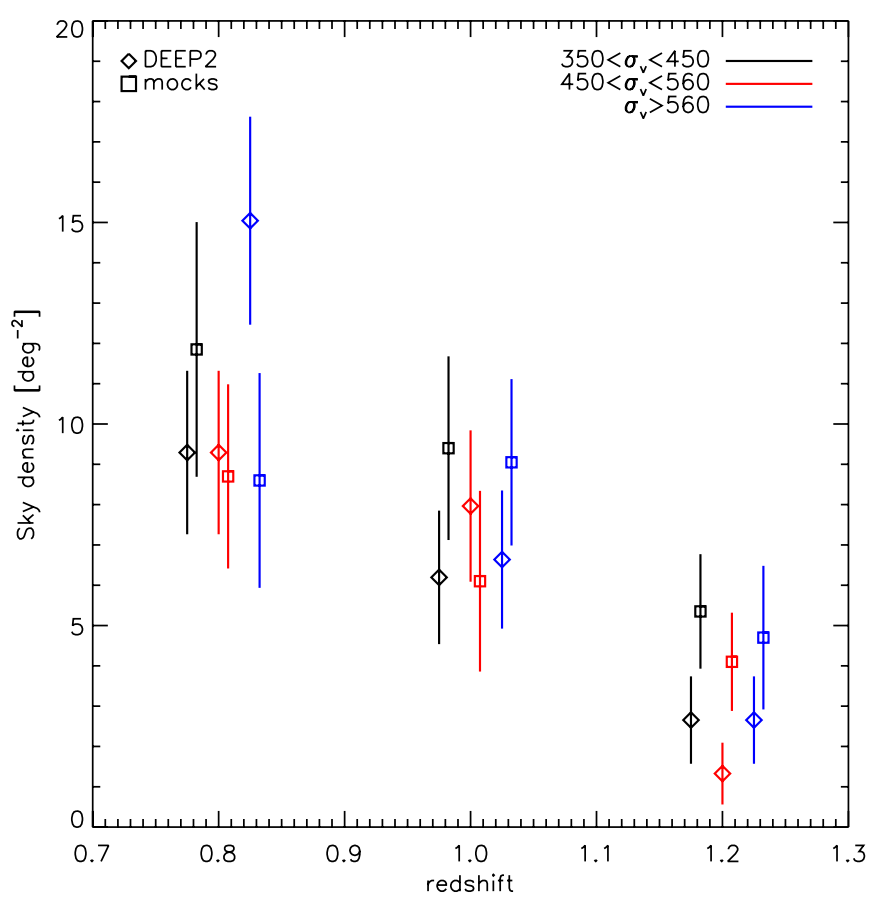

Figure 10. On-sky density of VDM galaxy groups in the DEEP2 survey and in the mocks, using the same redshift and velocity-dispersion bins that we used in Figure 4. DEEP2 points show the density of groups in the three high-redshift DEEP2 fields, and error bars show Poisson uncertainties on these measurements. Mock data points show the mean counts from 20 one-square-degree mock lightcones. Mock error bars show the estimated DEEP2 sample variance, which is computed as the standard deviation of the values from the 20 mocks reduced by a factor of 0.65 to account for the three independent DEEP2 fields, two of which are only partially observed.

(A color version of this figure is available in the online journal.)

of $\sigma_{\mathrm{v}}$ to the abundance of groups detected by VDM in the mocks. If we assume that the mocks are a sufficiently accurate representation of the galaxy population in groups, then this test constitutes a consistency check between DEEP2 and the background $\Lambda C D M$ cosmology of the Bolshoi mocks (which is very close to the current best fit to the available data). Figure 10 shows this comparison. Data points show the on-sky density of groups detected with VDM in DEEP2 and in the mocks, for groups with $\sigma_{\mathrm{v}} \geqslant 350 \mathrm{~km} \mathrm{~s}^{-1}$, using the same binning in redshift and $\sigma_{\mathrm{v}}$ that we did in Figure 4. The DEEP2 points are measured from the three high-redshift DEEP2 fields, and the error bars are Poisson uncertainties. The mock data points and error bars show the mean and standard deviation in the counts from 20 different mock lightcones, with the error bars reduced by a factor of 0.65 to account for the reduction in sample variance expected by combining the three independent high-redshift DEEP2 fields, given that two of them were only partially observed. We computed this factor using the samplevariance estimation techniques developed in Newman \& Davis (2000).

The mock and DEEP2 group abundances are in good statistical agreement at $z \lesssim 1$, suggesting that DEEP2 is in good agreement with the Bolshoi background cosmology and mock galaxy modeling in this regime. In the highest redshift bin, all three DEEP2 measurements lie below the mocks at roughly the $2 \sigma$ level. It is important to note that the three DEEP2 data points at each redshift are strongly correlated with one another, since the entire halo mass function will be scaled up or down in overdense or underdense regions of the universe. Hence, the similar disagreement for all three high-redshift $\sigma_{\mathrm{v}}$ bins does not 
necessarily indicate a systematic difference. We can conceive of three possible explanations for this discrepancy, which we list in decreasing order of plausibility: (1) the mock catalogs may not capture the DEEP 2 galaxy population at $z \gtrsim 1$ sufficiently accurately to model the group selection function, (2) the DEEP2 survey may probe a volume of the universe that is underdense at the $\sim 2 \sigma$ level at $z \gtrsim 1$, or (3) the growth of cosmic structure in the Bolshoi mocks is inconsistent with the real universe, indicating a problem with the current concordance cosmology. Construction of a DEEP 2 mock catalog that more accurately reproduces the measured two-point function would help to settle this question.

\section{REFERENCES}

Abell, G. O. 1958, ApJS, 3, 211

Adami, C., Durret, F., Benoist, C., et al. 2010, A\&A, 509, A81

Bardeen, J. M., Bond, J. R., Kaiser, N., \& Szalay, A. S. 1986, ApJ, 304, 15

Beers, B. C., Flynn, K., \& Gebhardt, K. 1990, AJ, 100, 32

Berlind, A. A., Frieman, J., Weinberg, D. H., et al. 2006, ApJS, 167, 1

Blaizot, J., Wadadekar, Y., Guiderdoni, B., et al. 2005, MNRAS, 360, 159

Butcher, H., \& Oemler, A. 1984, ApJ, 285, 426

Coil, A. L., Davis, M., Madgwick, D. S., et al. 2004a, ApJ, 609, 525

Coil, A. L., Gerke, B. F., Newman, J. A., et al. 2006a, ApJ, 638, 668

Coil, A. L., Newman, J. A., Cooper, M. C., et al. 2006b, ApJ, 644, 671

Coil, A. L., Newman, J. A., Kaiser, N., et al. 2004b, ApJ, 617, 765

Conroy, C., Wechsler, R. H., \& Kravtsov, A. V. 2006, ApJ, 647, 201

Cooper, M. C., Newman, J. A., Coil, A. L., et al. 2007, MNRAS, 376, 1445

Cooper, M. C., Newman, J. A., Croton, D. J., et al. 2006, MNRAS, 370, 198

Cucciati, O., Marinoni, C., Iovino, A., et al. 2010, A\&A, 520, A42

Davis, M., Gerke, B. F., \& Newman, J. A 2004, arXiv:astro-ph/0408344

Davis, M., Guhathakurta, P., Konidaris, N. P., et al. 2007, ApJ, 660, L1

De Lucia, G., \& Blaizot, J. 2007, MNRAS, 375, 2

Dressler, A. 1980, ApJ, 236, 351

Eke, V. R., Baugh, C. M., Cole, S., et al. 2004, MNRAS, 348, 866

Evrard, A. E., Bialek, J., Busha, M., et al. 2008, ApJ, 672, 122

Faber, S. M., Phillips, A. C., Kibrick, R. I., et al. 2003, Proc. SPIE, 4841, 1657

Faber, S. M., Willmer, C. N. A., Wolf, C., et al. 2007, ApJ, 665, 265

Fang, T., Gerke, B. F., Davis, D. S., et al. 2007, ApJ, 660, L27

Georgakakis, A., Gerke, B. F., Nandra, K., et al. 2008, MNRAS, 391, 183
Gerke, B. F., Newman, J. A., Davis, M., et al. 2005, ApJ, 625, 6

Gerke, B. F., Newman, J. A., Faber, S. M., et al. 2007, MNRAS, 376, 1425

Gladders, M. D., \& Yee, H. K. C. 2000, AJ, 120, 2148

Hao, J., McKay, T. A., Koester, B. P., et al. 2010, ApJS, 191, 254

Hu, W., \& Cohn, J. D. 2006, Phys. Rev. D, 73, 067301

Huchra, J. P., \& Geller, M. J. 1982, ApJ, 257, 423

Jeltema, T. E., Gerke, B. F., Laird, E. S., et al. 2009, MNRAS, 399, 715

Kenney, J. F. 1951, Mathematics of Statistics, Vol. 2 (Princeton, NJ: Van Nostrand-Reinhold)

Kitzbichler, M. G., \& White, S. D. M. 2007, MNRAS, 376, 2

Klypin, A., Trujillo-Gomez, S., \& Primack, J. 2011, ApJ, 740, 102

Knobel, C., Lilly, S. J., Iovino, A., et al. 2009, ApJ, 697, 1842

Koester, B. P., McKay, T. A., Annis, J., et al. 2007, ApJ, 660, 221

Le Fèvre, O., Vettolani, G., Garilli, B., et al. 2005, A\&A, 439, 845

Li, I. H., \& Yee, H. K. C. 2008, AJ, 135, 809

Lilly, S. J., Le Brun, V., Maier, C., et al. 2009, ApJS, 184, 218

Liu, H. B., Hsieh, B. C., Ho, P. T. P., Lin, L., \& Yan, R. 2008, ApJ, 681, 1046

Ma, C.-P., \& Fry, J. N. 2000, ApJ, 543, 503

Marinoni, C., Davis, M., Newman, J. A., \& Coil, A. L. 2002, ApJ, 580, 122

Milkeraitis, M., van Waerbeke, L., Heymans, C., et al. 2010, MNRAS, 406, 673

Miller, C. J., Nichol, R. C., Reichart, D., et al. 2005, AJ, 130, 968

Newman, J. A., \& Davis, M. 2000, ApJ, 534, 11

Newman, J. A., Marinoni, C., Coil, A. L., \& Davis, M. 2002, PASP, 114, 29

Newman, J. A., Cooper, M. C., Davis, M., et al. 2012, ApJ, submitted arXiv: 1203.3192

Nolthenius, R., \& White, S. D. M. 1987, MNRAS, 225, 505

Peacock, J. A., \& Smith, R. E. 2000, MNRAS, 318, 1144

Postman, M., Lubin, L. M., Gunn, J. E., et al. 1996, AJ, 111, 615

Press, W. H., \& Schechter, P. 1974, ApJ, 187, 425

Seljak, U. 2000, MNRAS, 318, 203

Sheth, R., \& Tormen, G. 2002, MNRAS, 329, 61

Soares-Santos, M., de Carvalho, R. R., Annis, J., et al. 2011, ApJ, 727, 45

Spitzer, L., \& Baade, W. 1951, ApJ, 115, 413

Springel, V., White, S. D. M., Jenkins, A., et al. 2005, Nature, 435, 629

Szapudi, I., \& Szalay, A. S. 1996, ApJ, 459, 504

Tinker, J., Kravtsov, A. V., Klypin, A., et al. 2008, ApJ, 688, 709

Vale, A., \& Ostriker, J. P. 2006, MNRAS, 371, 1173

Willmer, C. N. A., Faber, S. M., Koo, D. C., et al. 2006, ApJ, 647, 853

Yan, R., White, M., \& Coil, A. L. 2004, ApJ, 607, 739

Yang, X., Mo, H. J., van den Bosch, F. C., \& Jing, Y. P. 2005, MNRAS, 356, 1293

Zheng, Z., Berlind, A. A., Weinberg, D. H., et al. 2005, ApJ, 633, 791

Zheng, Z., Coil, A. L., \& Zehavi, I. 2007, ApJ, 667, 760 